\title{
Shape Effect on the Temperature Field during Microwave Heating Process
}

\author{
Zhijun Zhang $(\mathbb{D}$, Tianyi Su, and Shiwei Zhang $(\mathbb{D}$ \\ School of Mechanical Engineering and Automation, Northeastern University, Shenyang 110004, China \\ Correspondence should be addressed to Zhijun Zhang; zhjzhang@mail.neu.edu.cn
}

Received 9 November 2017; Revised 2 January 2018; Accepted 11 January 2018; Published 11 February 2018

Academic Editor: Hong-Wei Xiao

Copyright (C) 2018 Zhijun Zhang et al. This is an open access article distributed under the Creative Commons Attribution License, which permits unrestricted use, distribution, and reproduction in any medium, provided the original work is properly cited.

\begin{abstract}
Aiming at improving the food quality during microwave process, this article mainly focused on the numerical simulation of shape effect, which was evaluated by microwave power absorption capability and temperature distribution uniformity in a single sample heated in a domestic microwave oven. This article only took the electromagnetic field and heat conduction in solid into consideration. The Maxwell equations were used to calculate the distribution of microwave electromagnetic field distribution in the microwave cavity and samples; then the electromagnetic energy was coupled as the heat source in the heat conduction process in samples. Quantitatively, the power absorption capability and temperature distribution uniformity were, respectively, described by power absorption efficiency (PAE) and the statistical variation of coefficient (COV). In addition, we defined the comprehensive evaluation coefficient (CEC) to describe the usability of a specific sample. In accordance with volume or the wave numbers and penetration numbers in the radial and axial directions of samples, they can be classified into different groups. And according to the PAE, COV, and CEC value and the specific need of microwave process, an optimal sample shape and orientation could be decided.
\end{abstract}

\section{Introduction}

Microwave is a common process treatment of food products as an approach to accelerate the processes or improve the food quality. It can be used as a heat source in processes like heating [1-5], drying [6, 7], thawing, puffing [8,9], and so on; besides microwave can be also used as an assisting method in other processes like sterilization [10], moist measurement [11], and so on [12]. Compared with the conventional heating process microwave heating has many advantages, such as high heating efficiency, good uniformity, being controllable, easy maintenance, and being environment friendly $[13,14]$; thus microwave heating process has been applied in plenty of processes in food, nonmetal, and advanced materials industry since the 1950s. Although using microwave heating can achieve better uniformity compared with the conventional methods, hot spots and cold spots still occur in the sample, which seriously affect the quality of material during the process [15]. Improving temperature uniformity is still an urgent problem in microwave heating processes.

Microwave is an electromagnetic wave with a frequency range of $0.3 \mathrm{GHZ}$ to $300 \mathrm{GHZ}$, corresponding to the wavelength range of $0.001 \mathrm{~m}$ to $1 \mathrm{~m}$. The commonly used microwave frequencies are $0.915 \mathrm{GHZ}, 2.45 \mathrm{GHz}$, and $5.8 \mathrm{GHz}$, and most equipment designed for microwave processing is operated at $2.45 \mathrm{GHz}$ [16]. Electromagnetic field distribution of the microwave treatment process can be described by Maxwell's equations or Lambert's law [13]. The propagation and absorption of microwave electromagnetic field can be described by Maxwell's equations and the accuracy of Lambert's law which is based on an assumption of semi-infinite critical sample length [17], which is verified when the thickness of the sample is more than three times the microwave penetration depth of the sample [18]. Taking the scope of application and accuracy into account, Maxwell's equations are the best choice to describe the electromagnetic field distribution.

Materials placed in the microwave environment are classified as insulators, conductors, semiconductors, and superconductors [19]. Most of foodstuff are in the category of insulators and have low internal wavelength decided by dielectric properties and electromagnetic frequency [20]. Chemical component and physical structure of foodstuff determine the dielectric properties [13]. Food materials 
interact with microwave electromagnetic field to generate heat, and the dielectric constant and dielectric loss coefficient of the material are used to characterize the electromagnetic energy storage capability and the ability to convert electromagnetic energy into heat of the materials. So electromagnetic distribution can directly influence the temperature generation and distribution in the foodstuff heated by microwave [19]. Therefore, studying the rules from foodstuff heating phenomena in microwave oven has significant meaning for improving the quality of various foodstuff.

After the foodstuff treatment processes, the compositions of food product are main indicators to measure the quality. Enzymes, trace elements, proteins, and other substances in food are sensitive to temperature, and the high temperature during processing will destroy the food structure and even produce harmful substances, which seriously influences the food quality. Temperature nonuniformity in foodstuff leads to hot spots and cold spots. At the location of hot spots the temperature may be too high to damage the nutrient substance, and at the cold spots the low temperature may fail to reach the damage temperature of microorganisms, which raises the risk of food deterioration. Therefore, both hot spots and cold spots are drawbacks of the overall quality of the product [13]. In addition, compared with a single homogeneous material or synthetic material, foodstuff is more complex and anisotropic, so the interaction between microwave electromagnetic wave and food material becomes less uniform during the whole processing, which results in uneven distribution of temperature. Therefore, it is very important to study the temperature uniformity of food microwave processing.

Researches on foodstuff microwave treatment processes are mostly concentrated on two aspects: one aspect is the dielectric properties of the object itself, including factors affecting dielectric properties [18, 21], shape effect [22-24], and deformation effect [25]. Researches on aspects other than the target object like microwave equipment structure [26, 27], microwave properties [28], microwave processing strategies $[20,29]$, and experimental verifications $[30,31]$ are also being performed at the same time. Even though all factors affect the results in the microwave treatment process, it is too complex to take overall consideration of them. Therefore, researchers tend to select the dominant elements which may convert to each other to conduct studies. Foodstuff can be divided into four groups by the microwave heating characteristics, and potato can present the most commonly used vegetable materials [20] with a relatively complete system of characteristic parameters as well as representative properties $[15,16,20,21,23,32]$.

In this article, we studied the temperature field distribution of potato samples with different shapes, sizes, and position orientations using a domestic microwave oven with one $2.45 \mathrm{GHz}$ microwave source in TE10 mode rectangular waveguide in three dimensions. The basically physical model of the microwave oven is consistent with the Microwave Oven in COMSOL Multiphysics software, but the shape, size, and position orientations of potato samples change in a wide range. This article described and summed up the general rules of the sample microwave heating processing from the perspective of the interaction between microwave electromagnetic field and sample's dielectric properties. The temperature distribution of various samples at different times was intuitively drawn from the qualitative point of view. Quantitatively, the ratio of absorbed power and input power named power absorption efficiency (PAE) was used to describe the microwave energy absorption capability of samples, and the ratio of internal temperature standard deviation and average value, namely, coefficient of variation (COV) $[15,29,33]$, measured the temperature distribution uniformity of a sample at a given moment. A lower coefficient of variation $(\mathrm{COV})$ means a better internal temperature uniformity, and, in addition, defining the comprehensive evaluation coefficient (CEC) which was the ratio of power absorption efficiency to temperature COV value. The CEC value can measure the samples' usability at a certain condition, and a higher CEC value presents a sample with a high PAE and/or low COV, which could be chosen according to specific needs of microwave process.

\section{Materials and Methods}

2.1. Basic Assumption. In order to simplify the calculation process and save calculation time, the following assumptions were made for the simulation process within the allowable error range:

(i) The samples are isotropic homogeneous. The samples of different sizes or shapes can be considered as isotropic homogeneous.

(ii) The influence of mass transfer and phase transformation of the process is ignored. The aim of this study is to obtain the temperature field distribution during the initial stage sample, so the temperature is below the phase transition temperature at atmospheric pressure.

(iii) The dielectric constant and conductivity are constant. The dielectric properties of foodstuff are mainly affected by the moisture [25]. In this paper, the temperature is below the phase transition point, so the moisture content of the sample changed a little; thus the dielectric constant can be regarded as constant. Similarly, thermal conductivity of the sample is also regarded as a constant.

(iv) The heat transfer occurs only in the sample. Because of the shorter research time, and the thermal conductivity of the stationary air being smaller than the solid thermal conductivity, the heat transfer of the air in the microwave oven can be neglected in this study.

2.2. Physical Model. As shown in Figure 1, the physical model consisted of a microwave oven cavity, a waveguide, a glass tray, and a potato sample (with different shapes other than the one shown in Figure 1), and the cavity and rectangular waveguide were made of copper and the size of cavity was length $w_{O}=0.267 \mathrm{~m}$, width $d_{O}=0.27 \mathrm{~m}$, and height $h_{O}=$ $0.188 \mathrm{~m}$ and the rectangular waveguide was length $w_{g}=$ $0.05 \mathrm{~m}$, width $d_{g}=0.078 \mathrm{~m}$, and height $h_{g}=0.018 \mathrm{~m}$. Glass tray in the microwave electromagnetic field was regarded as transparent medium, and no heat was generated. In 


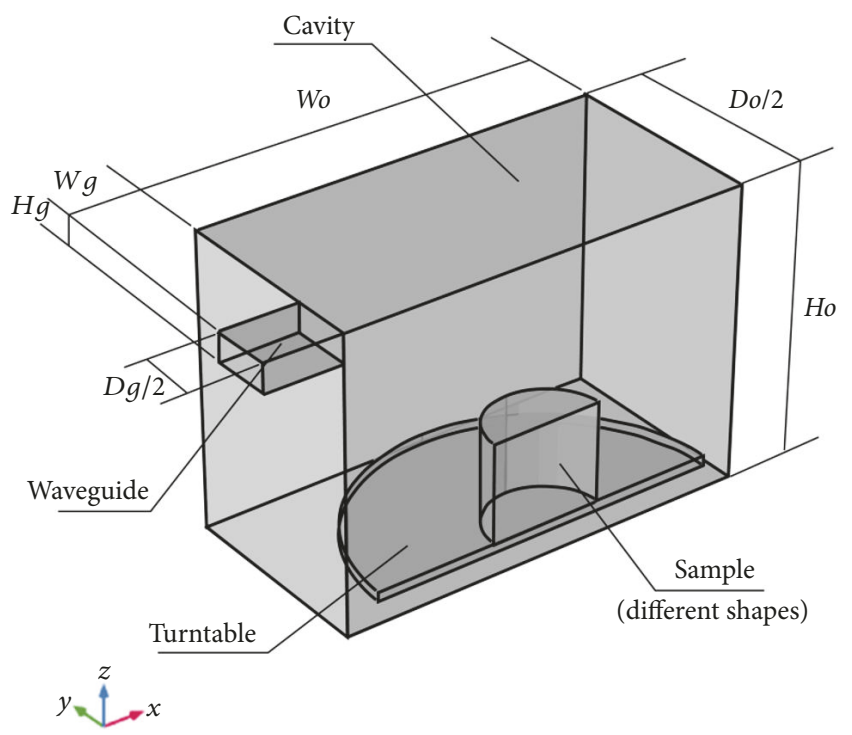

FIGURE 1: Schematic diagram of physical model.

order to reduce the amount of calculation and improve the computational efficiency, a symmetric model was established. The potato samples were in different shapes (sphere, ellipsoid, cylinder, cuboid, etc.), different sizes, and different position orientations.

\subsection{Mathematical Model}

2.3.1. Electromagnetic Field Model Establishment. The electromagnetic field distribution in the sample, microwave cavity, and waveguide was described by the Maxwell equations as follows:

$$
\begin{aligned}
& \nabla \cdot \vec{D}=\rho_{\text {ele }} \\
& \nabla \times \vec{E}=-\frac{\partial \vec{B}}{\partial t} \\
& \nabla \cdot \vec{B}=0 \\
& \nabla \times \vec{H}=\vec{j}+\frac{\partial \vec{D}}{\partial t},
\end{aligned}
$$

where $\vec{D}$ is the electric flux density, $\vec{E}$ is the electric field intensity, $\vec{B}$ is the magnetic flux density, $\vec{H}$ is the magnetic field intensity, $\rho_{\text {ele }}$ is the source of the electric field, and $\vec{j}$ is the current density.

The constitutive equations describing the interaction between material and electromagnetic wave in electromagnetic field were presented as follows:

$$
\begin{aligned}
& \vec{D}=\varepsilon_{0} \varepsilon_{r} \cdot \vec{E} \\
& \vec{B}=\mu_{0} \mu_{r} \cdot \vec{H} \\
& \vec{j}=\sigma \cdot \vec{E},
\end{aligned}
$$

where $\varepsilon_{r}$ is the relative permittivity, $\mu_{r}$ is the relative permeability, $\sigma$ is the conductivity, $\varepsilon_{0}$ is the permittivity of free space $\left(\varepsilon_{0}=8.854 \times 10^{-12} \mathrm{~F} / \mathrm{m}\right)$, and $\mu_{0}$ is the permeability of free space $\left(\mu_{0}=4 \pi \times 10^{-7} \mathrm{H} / \mathrm{m}\right)$.

In the physical model, no conduction current existed in the microwave cavity and sample, and both of the electric and magnetic field were passive field; thus in this case the following can be concluded: $\rho_{\text {ele }}=0, \vec{j}=0$. The wave equation in microwave cavity was as follows:

$$
\nabla \times \mu_{r}^{-1}(\nabla \times E)-k_{0}^{2} \varepsilon_{r} E=0,
$$

where $k_{0}$ is electromagnetic wave in free space, described as

$$
k_{0}=\omega \sqrt{\varepsilon_{0} \mu_{0}} .
$$

2.3.2. Heat Transfer Model Establishment. The samples were treated as solid, interacted with electric field, and generated heat in the food interior. The electromagnetic heat then transferred to the low temperature part in the way of conduction. The heat transferred ability of the sample and its surrounding environment was low because of still air around the sample. Therefore, only heat conduction in the sample was considered, and that in the surrounding environment was neglected. Besides, water evaporation of the sample was also neglected due to the low water evaporation rate in the period before the phase transition temperature of the hot spot. The microwave electromagnetic energy was used as heat source to couple the electromagnetic energy with the energy equation. The heat transfer process was described as follows [29, 34]:

$$
\begin{aligned}
\rho C_{p} \frac{\partial T}{\partial t} & =\nabla \cdot(k \nabla T)+Q_{\text {mic }} \\
Q_{\text {mic }} & =\frac{1}{2} \omega \varepsilon_{o} \varepsilon^{\prime \prime}|E|^{2},
\end{aligned}
$$

where $\rho$ is the density of the sample, $C_{p}$ is the specific heat capacity, $k$ is the thermal conductivity of the sample, $T$ is the temperature, $Q_{\text {mic }}$ is the microwave heat source, and $\omega$ is the angular frequency of microwave.

2.3.3. Initial Condition. Initial values of electromagnetic field components were all zero except the initial value of $z$ directional electric field component, which was described as follows:

$$
E_{z 0}=\cos \frac{\pi y}{d g} .
$$

Besides, initial ambient temperature was $T_{\text {atm } 0}=20^{\circ} \mathrm{C}$, and the initial temperature of sample was $T_{0}=8^{\circ} \mathrm{C}$.

2.3.4. Boundary Condition. The cavity and waveguide were considered as the copper wall resistance loss, using impedance boundary conditions to define the wall, described as follows:

$$
\begin{aligned}
& \sqrt{\frac{\mu_{0} \mu_{r}}{\varepsilon_{0} \varepsilon_{r}-j(\sigma / \omega)}} n \times H+E-(n \cdot E) n \\
& =\left(n \cdot E_{s}\right) n-E_{s} .
\end{aligned}
$$


TABLE 1: Physical parameters of potato samples.

\begin{tabular}{lccc}
\hline Symbol & Value & Unit & Description \\
\hline$\varepsilon^{\prime}$ & 58 & 1 & Dielectric constant \\
$\varepsilon^{\prime \prime}$ & 13 & 1 & Dielectric loss coefficient \\
$\mu_{r}$ & 1 & 1 & Magnetic permeability \\
$\sigma$ & 0 & $\mathrm{~S} / \mathrm{m}$ & Conductivity \\
$k$ & 0.648 & $\mathrm{~W} /(\mathrm{m} \cdot \mathrm{K})$ & Thermal conductivity \\
$\rho$ & 1067 & $\mathrm{~kg} / \mathrm{m}^{3}$ & Density \\
$C_{p}$ & 3630 & $J /(\mathrm{kg} \cdot \mathrm{K})$ & Specific heat capacity \\
\hline
\end{tabular}

The plane of symmetry used electrical conductor boundary conditions to eliminate the influence on the imaginary plane of the simulation process, by the following formula:

$$
n \times H=0 .
$$

The propagation constant of electromagnetic field was expressed by

$$
\beta=\frac{2 \pi}{c} \sqrt{f^{2}-\frac{c^{2}}{4 d g^{2}}}
$$

The whole simulation process was carried out under atmospheric pressure, and the pressure boundary conditions were set to $1 \mathrm{~atm}$.

2.3.5. Sample Parameters. The sample was potato, and the related parameters of the sample were shown in Table 1 [35].

\section{Results and Discussion}

In order to verify the accuracy of the mathematical model of this article, this model was used to calculate the physical model in [27]; the specific calculation process was not expressed in this article. The calculated results were compared with the results in [27], as shown in Figure 2. It can be seen from the diagram that the results obtained by the mathematical model in this paper are close to the results in the literature, but the temperature rise rate is slightly larger than that in literature. Therefore, the mathematical model selected in this paper could basically meet the needs of the research.

In Figure 3, there were several typical temperature distribution results of potato examples with same calculation conditions except for shape or orientation. It can be clearly seen in the figures that examples with different shapes or orientations formed some hot spots in different position in different time. Obviously, the hot spots must be the first positions to reach the phase transition temperature, and at the same time temperature at other positions of the food sample would be lower than that of hot spots. In Figure 3, the deeper red color indicated the lower temperature, and on the contrary the brighter yellow color indicated the higher temperature. Meanwhile, the images in the same row showed the whole heating process from the beginning to the time that the maximum temperature of the hot spots reached the

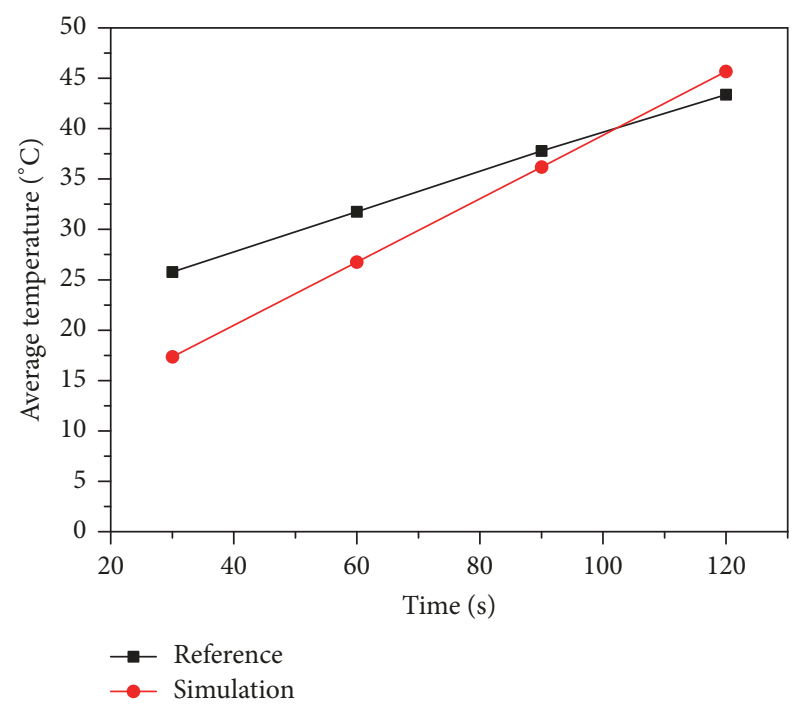

Figure 2: Model validation.

phase transition temperature. It is not difficult to conclude from Figure 3 that when the hot spots reach the phase transition temperature, the internal temperature distribution of the potato samples changes with shape or orientation of the sample. For the microwave treatment process of different samples, a process with good uniformity, high absorption power efficiency, small temperature difference, and short time is expected. Therefore, it is of great practical significance to study the influence of sample shape on the initial temperature field establishment of microwave process.

3.1. Microwave Power Input Effect. Heating power is the energy applied to the samples, partially producing dielectric heating effect [36]. The increasing or decreasing of input power directly affects the heat generated in the sample per unit time, then the heating rate of the sample is affected as well. The hot spots formed in the sample, as shown in Figure 3, would firstly reach the boiling point at each input power. There were some microwave power distribution grayscale color map images of the elliptical sample in reference [22], which was corresponding to the hot spots in the ellipsoid shaped samples in 2D. Studies focused on the period before the phase transition temperature was conducted and obtained the temperature field establishment process.

From Figure 4 it can be seen that the maximum temperature within a cylinder sample with radius $0.016 \mathrm{~m}$ and height $0.05 \mathrm{~m}$ reached the phase transition temperature after being heated by different input power within $200 \mathrm{~s}$ (except for $10 \mathrm{~W})$. The greater the input power, the shorter the time it takes to reach the phase transition temperature. The PAE of microwave power is about 0.63 and remains constant during the process due to the constant dielectric loss coefficient of the sample and constant input power under the specific study condition. Therefore, before the hot spots reach the phase transition temperature, input power of the microwave has little effect on PAE of the potato samples. The direct effect of the input power is the time it spent on reaching the phase 


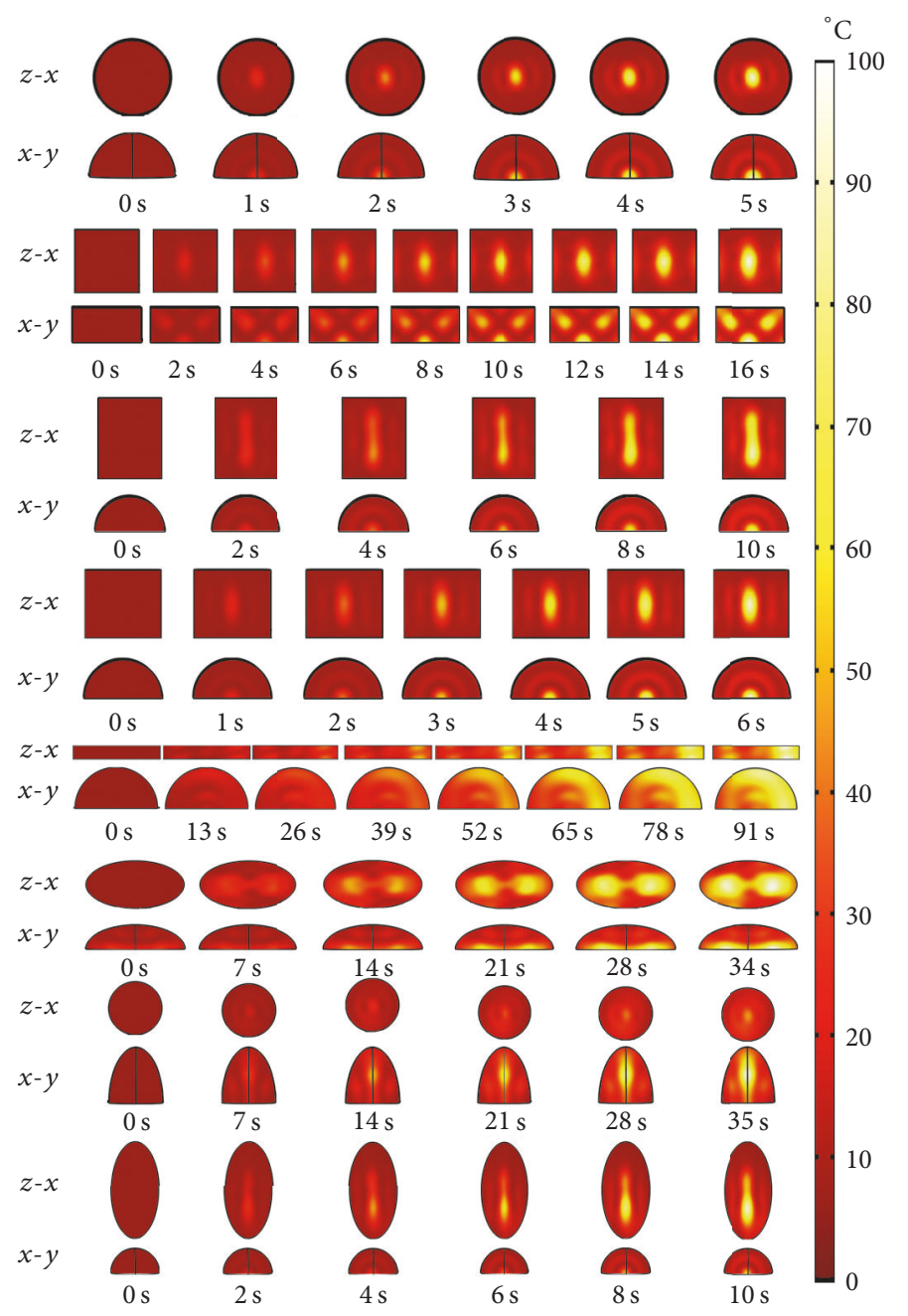

FIGURE 3: Hot spot in different samples.

transition temperature as shown in Figure 4; this is consistent with [30].

As we all know, energy consumption is an important measurement to a productive process, so the total energy of different input power consumed before the phase transition temperature should be considered to choose an optimal input power. As the curve has shown in Figure 5, it indicates that when the internal hot spots reach the phase transition temperature, the total energy consumption decreases from $4116 \mathrm{~J}$ to $630 \mathrm{~J}$ corresponding to the input power increasing from $10 \mathrm{~W}$ to $200 \mathrm{~W}$. Besides, when the input power is larger than $80 \mathrm{~W}$, the total energy consumption tends to be stable from the curve in Figure 5. Taking the previous work into consideration, it suggests that properly increasing input power will reduce energy consumption.

Figures 6 and 7 show the potato samples' temperature uniformity. In Figure 6, curves indicate the COV time variation of different input power before the internal hot spots reach the phase transition temperature (except $10 \mathrm{~W}$ ). It can be grasped that COV history of the sample internal temperature distribution tends to be level-off for the relatively lower input power but increases linearly for the higher ones; both of the phenomena can be seen in [29]. For the lower input power, it takes a relatively long time to reach the phase transition temperature, and during this period heat generated in the penetration depth is conducted to the lower temperature locations, which makes a positive effect of the temperature uniformity. As for the higher input power, hot spots reach the phase transition temperature in a short time, during which the heat generated in the penetration depth cannot be adequately conducted to the lower temperature locations; as a consequence the temperature uniformity is worse under these circumstances.

The final COV-input power curve at the hot spots phase transition moment can be seen in Figure 7. It demonstrates that when input power is larger than $80 \mathrm{~W}$, the final COV remains about 0.5 as the input power rises, while when the input power is less than $80 \mathrm{~W}$, the final COV increases from about 0.3 to 0.5 as the input power rises. The reason why the final COV tends to be stable can be attributed to same penetration depth [24] and short time before the hot spots phase transition moment. From the equations in [24], the penetration depth of a microwave process depends on the microwave frequency and the sample's dielectric properties 


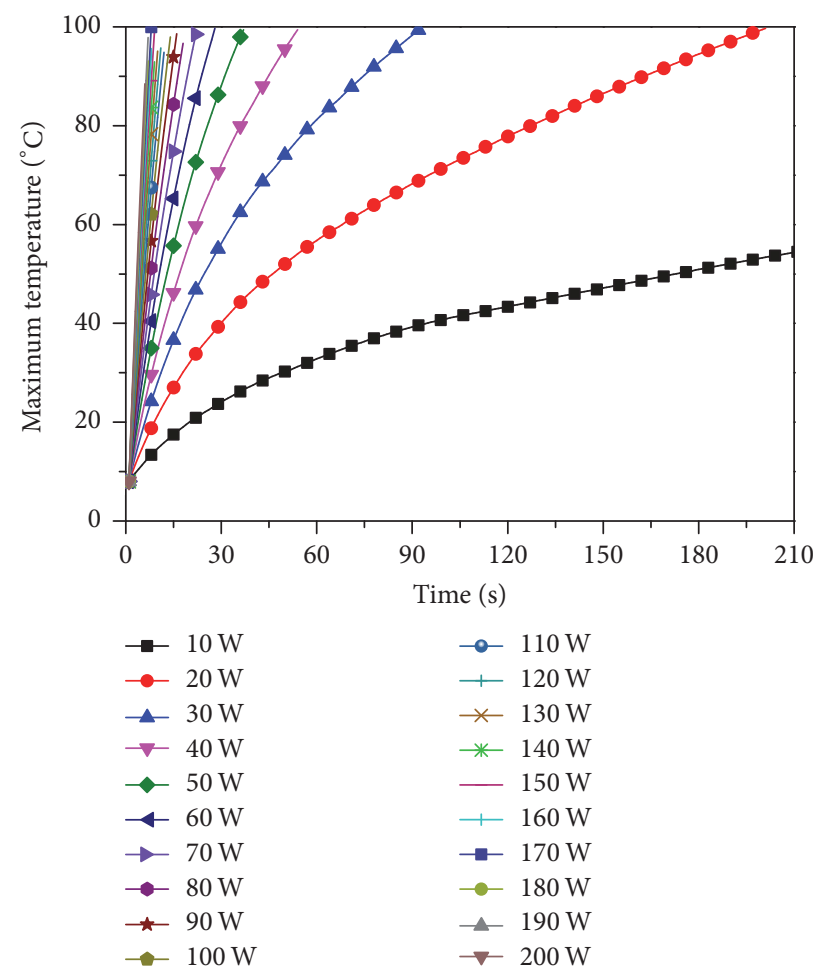

FIgURE 4: Maximum temperature of different input power.

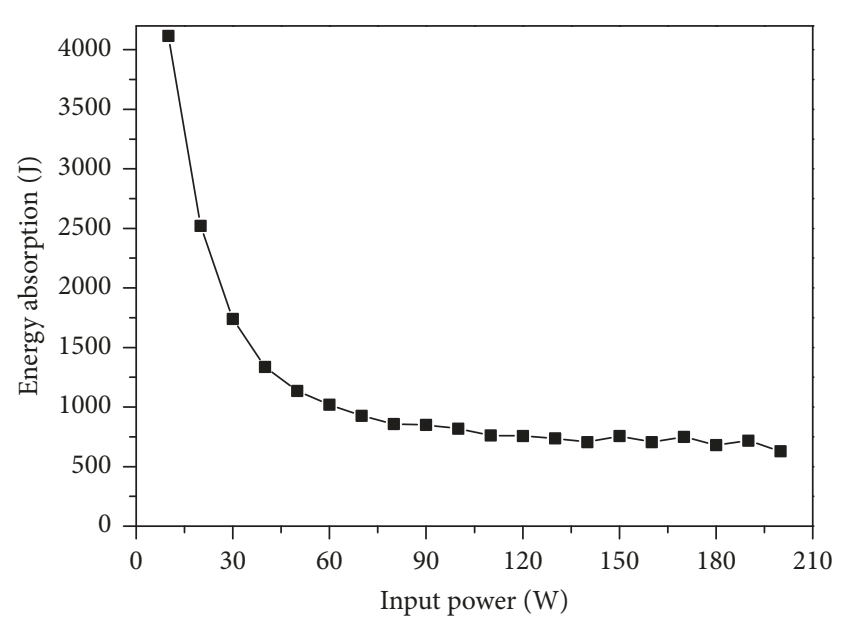

FIGURE 5: Energy consumption of different input power.

which in our study remain constant, so it can be concluded that during the microwave process in this case the heat generated in the penetration depth of the potato sample remains constant, too. Besides, when input power is larger than $80 \mathrm{~W}$, the time for rising temperature is shorter than that used to heat conduction, so the heat generated almost stays within the area of penetration depth. Thus, the COV values of internal temperature distribution stay constant. From Figure 7, it can be also concluded that lower input power can make a better temperature uniformity, and with the increasing of input power, the uniformity of temperature

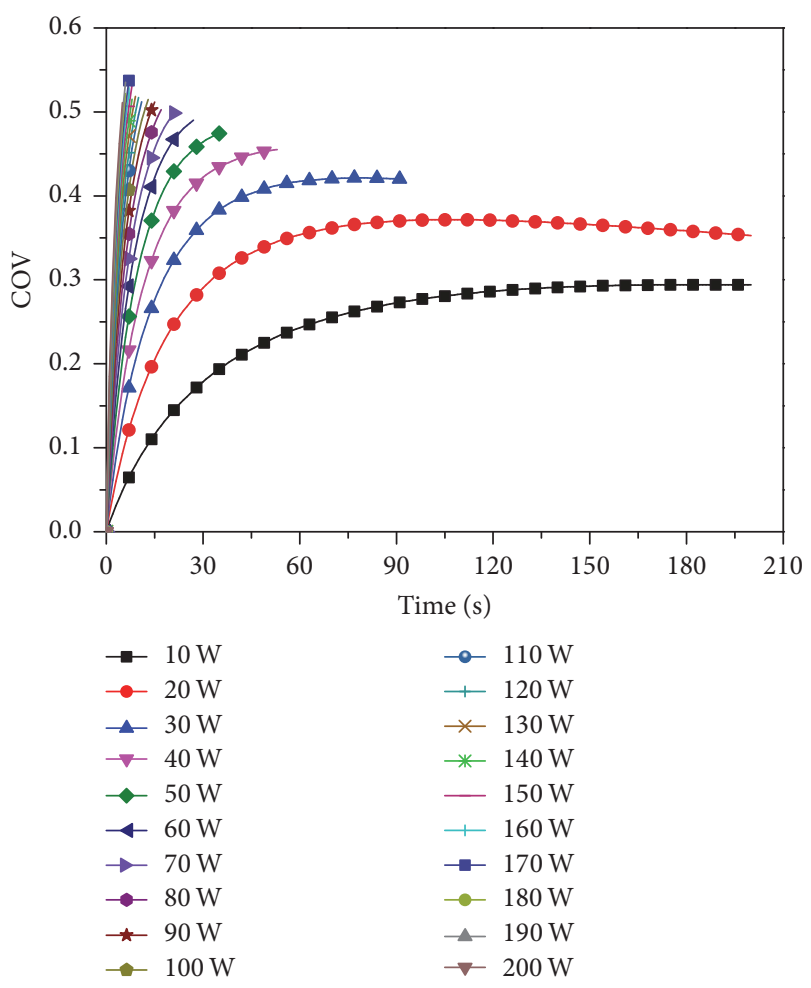

FIgURE 6: Time variation of temperature uniformity.

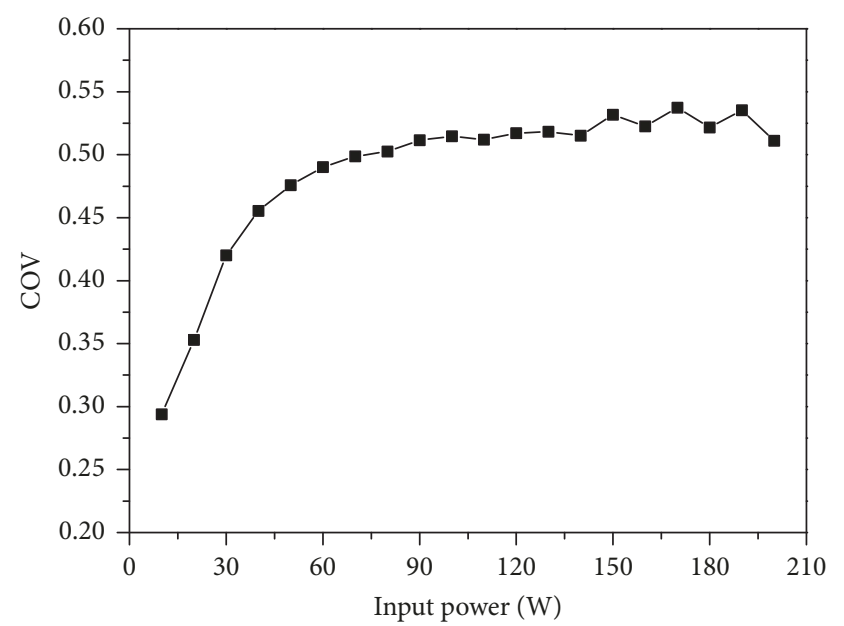

FIgURE 7: Temperature uniformity of different input power.

becomes worse, the same conclusion was drawn in reference [30].

To sum up, relatively low input power leads to a more uniform temperature distribution in potato samples, but excessive pursuit of uniform temperature reduces heating efficiency and increases energy consumption. Relatively high input power can dramatically shorten heating time and decrease energy consumption, but the uniformity in potato samples at the phase transition time becomes worse. Therefore, in order to consume less energy and get more uniform temperature distribution, it is necessary to choose a proper input power for specific samples. 
TABLE 2: Geometric characteristics dimension of different shape potato samples.

\begin{tabular}{lcccc}
\hline Number & Shape & \multicolumn{2}{c}{ Characteristics dimension $\left(10^{-3} \mathrm{~m}\right)$} & $\mathrm{V} 3\left(\mathrm{~m}^{3}\right)=13.11 \times 10^{-5}$ \\
\hline$(1)$ & Sph. & $\mathrm{V} 1\left(\mathrm{~m}^{3}\right)=1.64 \times 10^{-5}$ & $R 31.018$ & $R 39.702$ \\
$(2)$ & Cub. & $\mathrm{L} 23.851$ & $\left.\mathrm{~L} \mathrm{~m}^{3}\right)=6.25 \times 10^{-5}$ & $\mathrm{~L} 64$ \\
$(3)$ & Cyl.1 & $R 16-H 40.7$ & $R 16-H 155.4$ & $R 25-H 133.5$ \\
$(4)$ & Cyl.2 & $R 25-H 16.7$ & $R 25-H 63.7$ & $R 32-H 81.5$ \\
$(5)$ & Cyl.3 & $R 32-H 10.2$ & $R 32-H 38.9$ & $R 50-H 33.4$ \\
$(6)$ & Cyl.4 & $R 50-H 4.2$ & $R 50-H 15.9$ & $R 64-H 20.4$ \\
$(7)$ & Cyl.5 & $R 64-H 2.5$ & $R 64-H 9.7$ & $x 32-y z 44.2$ \\
$(8)$ & Ell.1 & $x 32-y z 15.6$ & $x 32-y z 30.5$ & $y 32-x z 44.2$ \\
$(9)$ & Ell.2 & $y 32-x z 15.6$ & $y 32-x z 30.5$ & $z 32-x y 44.2$ \\
$(10)$ & Ell.3 & $z 32-x y 15.6$ & $z 32-x y 30.5$ & $x 50-y z 35.4$ \\
$(11)$ & Ell.4 & $x 50-y z 12.5$ & $x 50-y z 24.4$ & $y 50-x z 35.4$ \\
$(12)$ & Ell.5 & $y 50-x z 12.5$ & $y 50-x z 24.4$ & $z 50-x y 35.4$ \\
$(13)$ & Ell.6 & $z 50-x y 12.5$ & $z 50-x y 24.4$ & $x 64-y z 31.3$ \\
$(14)$ & Ell.7 & $x 64-y z 11.1$ & $x 64-y z 21.6$ & $y 64-x z 31.3$ \\
$(15)$ & Ell.8 & $y 64-x z 11.1$ & $y 64-x z 21.6$ & $z 64-x y 31.3$ \\
$(16)$ & Ell.9 & $z 64-x y 11.1$ & $z 64-x y 21.6$ & \\
\hline
\end{tabular}

3.2. Sample Shapes Effect. In microwave processing various shapes of samples may be dealt with. This part mainly focused on the samples' shape effect on microwave power absorption capability and temperature distribution uniformity of potato samples before the internal hot spots reach the phase transition temperature. The sample shape can be simplified as sphere, cube, cylinder, ellipsoid, and cuboid. In the course of this section, except for the sample shape, all properties including physical and geometric properties of the sample were set to be the same. Input power was $90 \mathrm{~W}$. There were three groups of samples classified by volume, as shown in Table 2 (the blank indicates the characteristics size of the sample is beyond the computational domain). These samples were placed in the middle of the glass tray. Samples number 1 and number 2 are sphere and cube, which have only one characteristics dimension. The samples who have more than one characteristics dimension, namely, number 3 to number 7 , are cylindrical samples with increasing radius and decreasing height, and number 8 to number 16 are three groups of ellipsoidal samples placed in different axis directions. When the internal hot spots reached the phase transition temperature, samples' power absorption efficiency $(\mathrm{PAE})$ and temperature distribution uniformity $(\mathrm{COV})$ were shown in Figures 8 and 9, respectively.

In Figures 8 and 9, it can be clearly seen that the PAE and COV values of spherical samples are less than those of isometric volume cubic samples. In detail, the sphere samples' PAE of V1, V2, and V3 are $0.78,0.65$, and 0.67 , respectively, which are less than those of corresponding cubic samples 0.81 , 0.77 , and 0.81 . Similarly, the sphere samples' COV values of $\mathrm{V} 1, \mathrm{~V} 2$, and $\mathrm{V} 3$ are $0.45,0.42$, and 0.47 , respectively, which are less than those of cubic samples $0.50,0.56$, and 0.56 . Therefore, there is an enhancement of power absorption capability and a detriment of temperature distribution uniformity for cubic samples in contrast to spherical ones. The differences

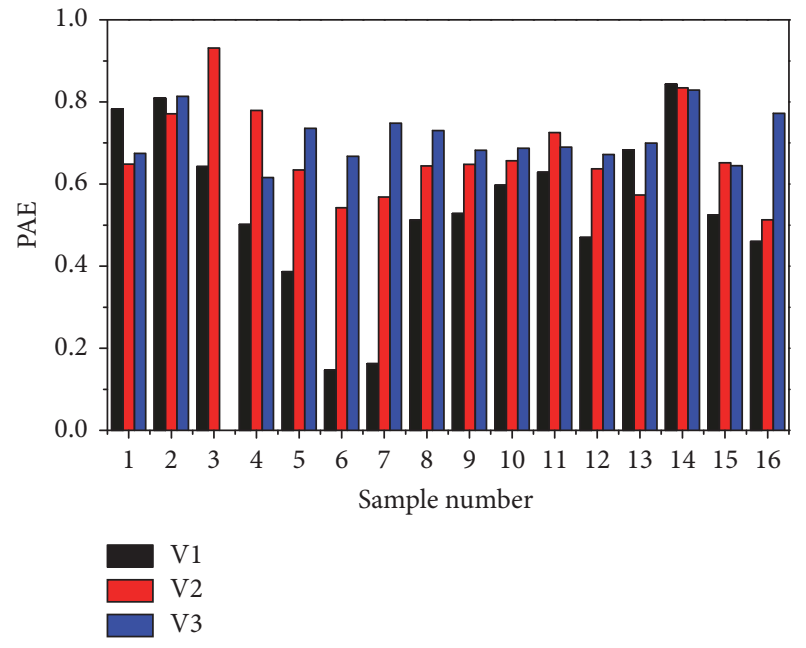

Figure 8: PAE of different shape.

between spherical and cubic samples on power absorption capability and temperature distribution uniformity mainly originate from the focusing tendency of microwave at the samples' corners and were concluded in the two-dimension simulation of reference [37] and can be also seen in the result diagram of cubic sample in Figure 3. Further comparison between spherical and cubic samples will be discussed in Section 3.2.1.

Contrasting the PAE and COV values of number 3 to number 7 isometric volume cylindrical samples in Figures 8 and 9 , an obvious fluctuation with the increasing radius and decreasing height can be seen, but the position of extreme values is unfixed for different volume sample groups. Similar phenomena can be seen by contrasting the samples of number 8 , number 11, and number 14 (similar comparisons 

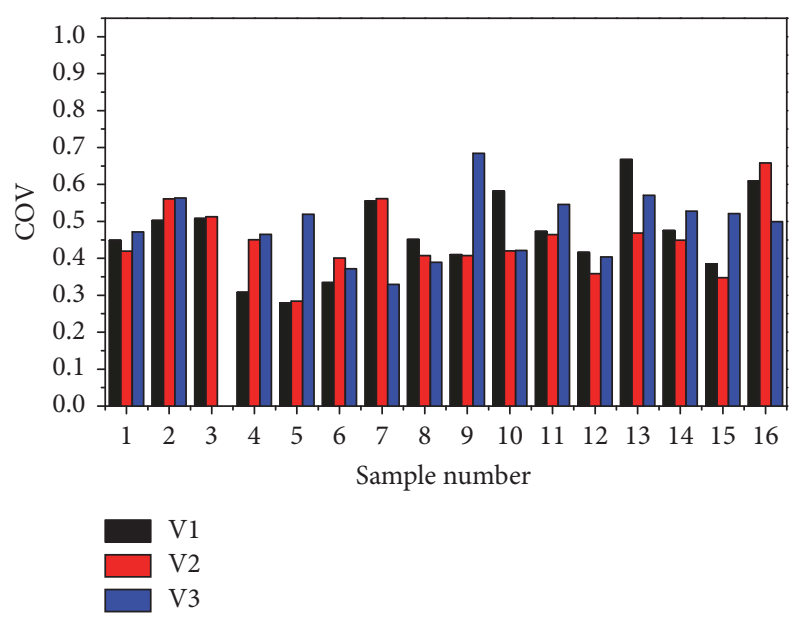

Figure 9: COV of different shape samples.

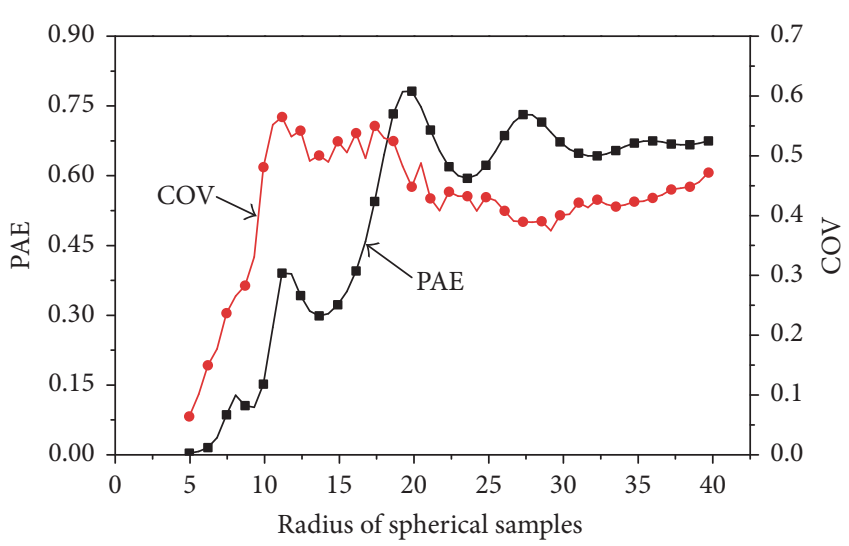

Figure 10: PAE and COV of sphere.

are number 9 , number 12 , and number 15 , or number 10 , number 13, and number 16). It will be discussed further in Section 3.2.2.

By contrasting the PAE and COV values of number 8 to number 10 samples (similar comparisons are number 11 to number 13 , or number 14 to number 16 ), there are obvious changes of different placement directions, and the variation tendency may increase, wave, or decrease. Therefore, being treated in a single port microwave oven, placement direction of a sample has also a significant effect on samples' power absorption capability and temperature distribution uniformity, which will be discussed in Section 3.3.

3.2.1. Spherical and Cubic Sample. In this section, a comparative study of spherical and cubic samples was conducted. Spherical samples' radii were from $0.005 \mathrm{~m}$ to $0.04 \mathrm{~m}$, and the corresponding isometric volume cubic samples' side length were from $0.008 \mathrm{~m}$ to $0.064 \mathrm{~m}$ at an interval of $0.001 \mathrm{~m}$, and the isometric volume samples were numbered from number 1 to number 57. The input power was $90 \mathrm{~W}$, and the two groups of samples were heated to the moment when the temperature of the internal hot spots reached phase transition temperature. Figures 10 and 11 presented the PAE and COV

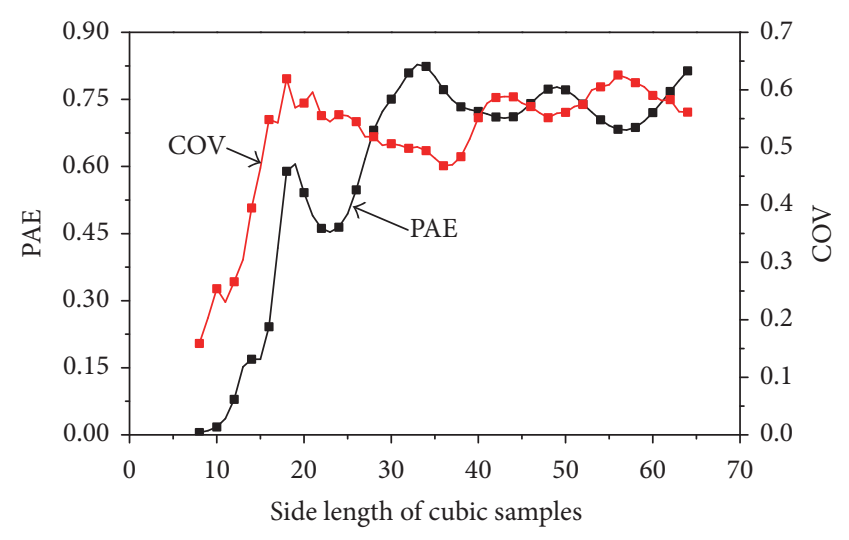

FIGURE 11: PAE and COV of cube.

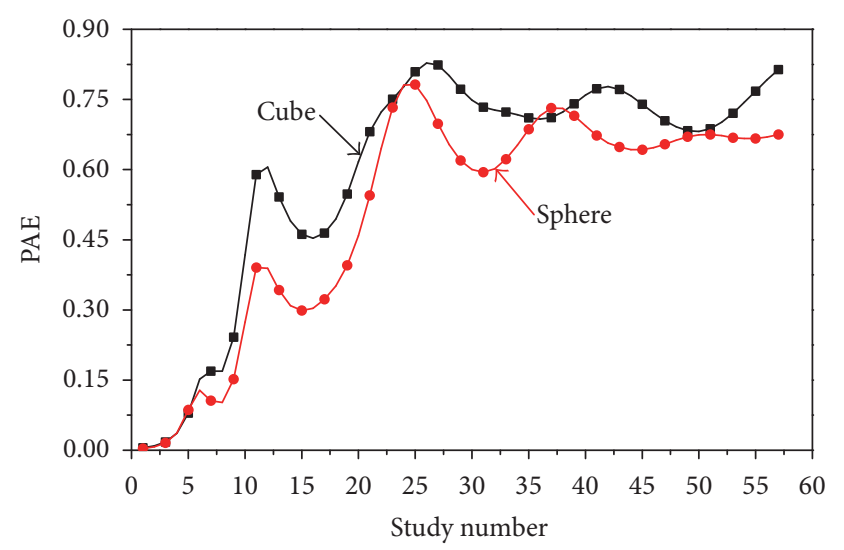

FIGURE 12: PAE comparison of cubic and spherical sample.

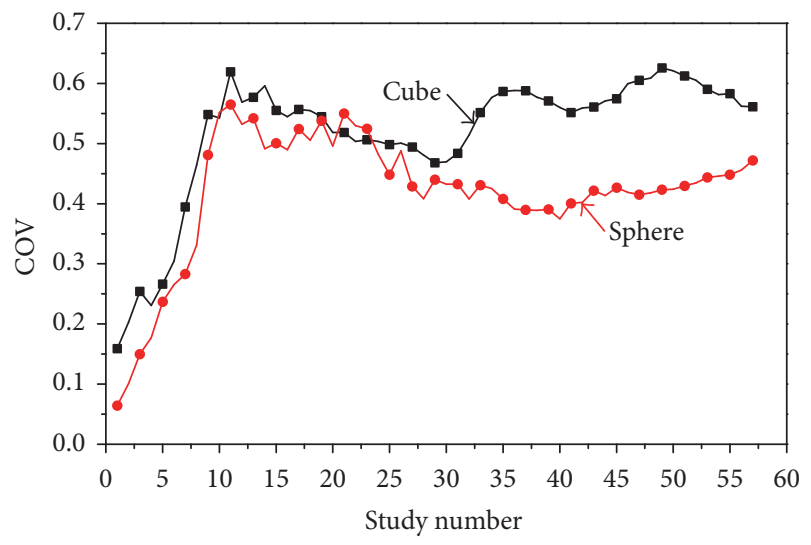

FIgURE 13: COV comparison of cubic and spherical sample.

values of the two series of samples, separately. PAE and COV values comparisons between cubic and spherical samples are shown in Figures 12 and 13. Figures 14 and 15 indicate some typical intuitive calculation results of electromagnetic field and temperature distribution.

The black curve in Figure 10 indicates the PAE values of spherical samples with increasing radii. It can be clearly seen that small radii spherical samples' capacity of absorbing 


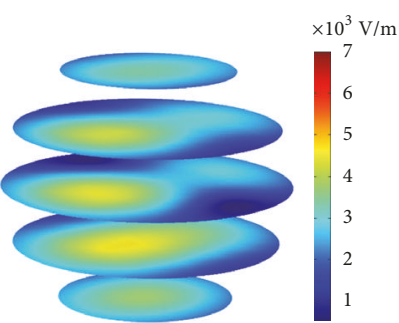

(a) $0.017 \mathrm{~m}$ diameter

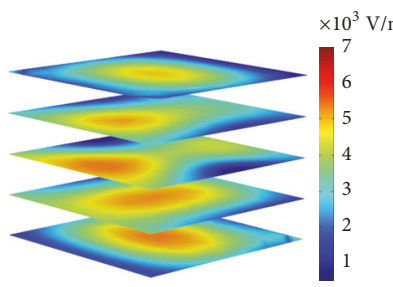

(e) $0.014 \mathrm{~m}$ side length

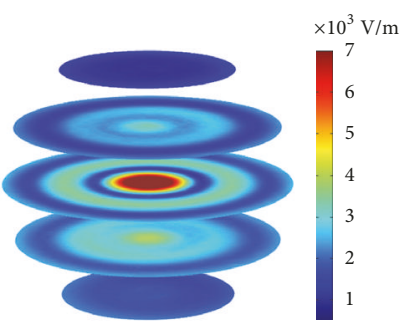

(b) $0.037 \mathrm{~m}$ diameter

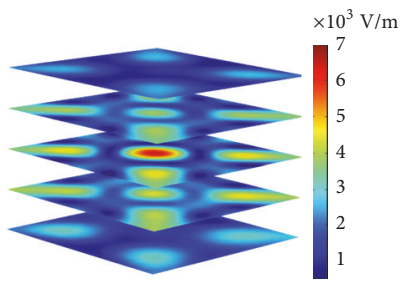

(f) $0.030 \mathrm{~m}$ side length

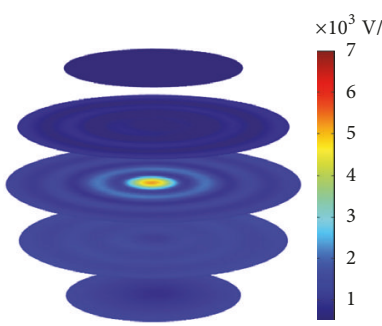

(c) $0.058 \mathrm{~m}$ diameter

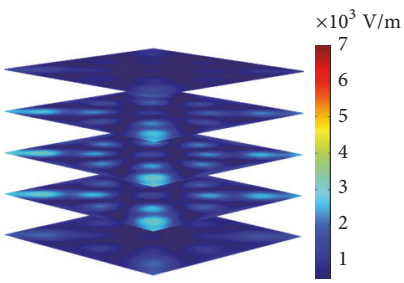

(g) $0.047 \mathrm{~m}$ side length

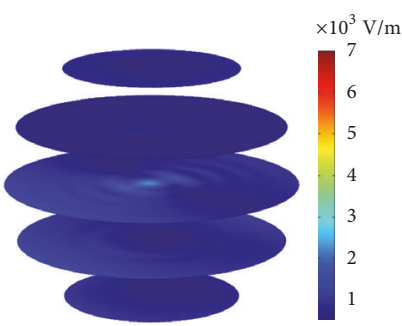

(d) $0.079 \mathrm{~m}$ diameter

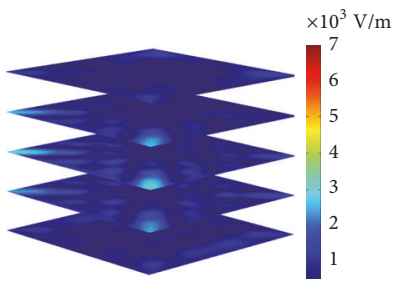

(h) $0.064 \mathrm{~m}$ side length

FIGURE 14: Spatial distribution of electromagnetic field in samples.

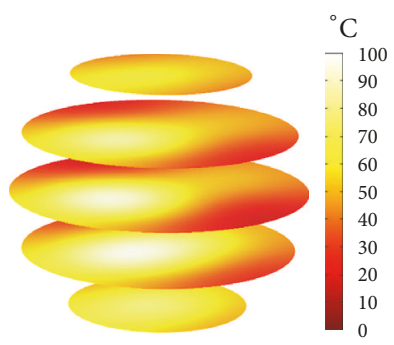

(a) $0.017 \mathrm{~m}$ diameter

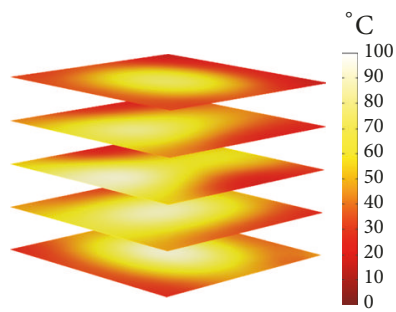

(e) $0.014 \mathrm{~m}$ side length

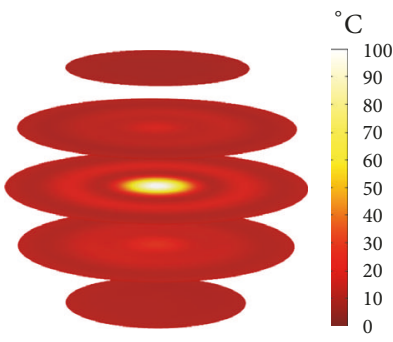

(b) $0.037 \mathrm{~m}$ diameter

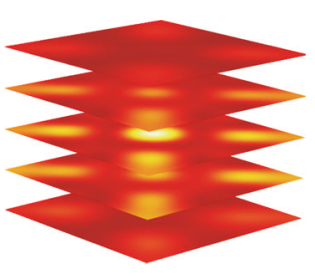

(f) $0.030 \mathrm{~m}$ side length

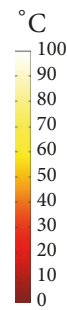

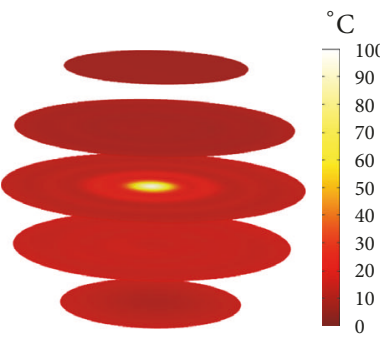

(c) $0.058 \mathrm{~m}$ diameter

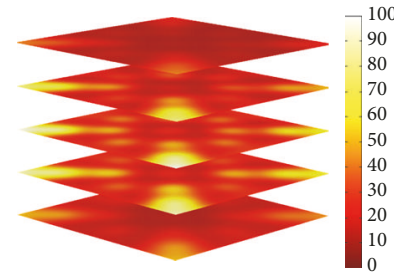

(g) $0.047 \mathrm{~m}$ side length

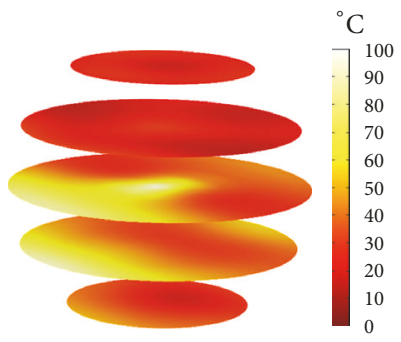

(d) $0.079 \mathrm{~m}$ diameter

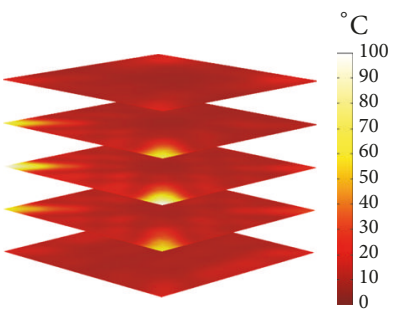

(h) $0.064 \mathrm{~m}$ side length

FIGURE 15: Spatial distribution of temperature in samples.

energy is low in contrast to the larger ones, and the PAE values of spherical samples increase as the radii rise. These phenomena were also shown in [38]. The lower PAE values of small spherical samples are resulting from the low temperature rising rate of these samples and lead to a better temperature distribution uniformity in the smaller samples. Likewise, higher PAE values of larger spherical samples would lead to worse temperature uniformity. Therefore, the red curve in Figure 10 represents the COV values being lower in the small samples and rises to higher values when the radii increase.

Interestingly, by contrasting the two curves in Figure 10, when spherical samples' radii increase, the COV curve firstly reaches an extreme value when the corresponding PAE value is still at a lower position. This maxima of COV values resulted from the focusing effect at internal local positions of samples [38]. Then when the samples' volume becomes large, the PAE curve stays stable, while the COV curve tends to increase. This phenomenon is due to the samples' large size which leads to heating only near the surface [38]. For the cubic samples' power absorption efficiency and COV curves in Figure 11, we can also find similar phenomena to the previous study of spherical samples. So phenomena in Figure 11 can be explained by the previous theory as well.

By contrasting the corresponding curve values of these two kinds of samples shown in Figures 12 and 13, there exist enormous differences caused by shape. Figure 12 shows the PAE values of equal volume cubic and spherical samples; it 
can be directly seen that most of the red curve is below the black one which means that in most cases cubic samples' capacity of absorbing microwave power is better than that of spherical ones. Similarly in Figure 13 the red curve is below the black one at most locations, which indicates that in most cases the temperature distribution uniformity of spherical samples is better than cubic samples.

Quantitatively, the PAE values of spherical samples range from 0.004 to 0.782 , while those of cubic samples range from 0.005 to 0.828 . Only for number 4 , number 5 , number 24, and number 36 to number 38 studies, cubic samples' values are lower than the spherical ones. Meanwhile, the COV values of spherical samples range from 0.064 to 0.565 , and those of cubic samples range from 0.159 to 0.626 . Only for number 10 and number 21 to number 23 studies, cubic samples' COV values are lower than the spherical ones. Combining with the above two phenomena, for number 4 , number 5, number 24, and number 36 to number 38 studies, spherical samples are better than cubic ones due to the high PAE and low temperature COV, and for number 10 , and number 21 to number 23 studies, cubic samples are better than spherical ones because of the same reason. Except for the above samples the cubic samples all have higher PAE and higher temperature COV values, which means a better power absorption capability and worse temperature distribution uniformity. Therefore, in most cases when a microwave process needs a high PAE, cubic sample is better, while if temperature uniformity is more important, it is recommended to choose spherical samples.

For example, choosing four sizes that represent the small, intermediate, and large samples (number 7, number 23, number 40, and number 57 samples) from the spherical samples and the corresponding cubic samples, Figures 14 and 15 show the spatial distribution of electromagnetic field and temperature of the four kinds of samples. Results shown in Figures 14(a)-14(d) and 15(a)-15(d) are quite similar with that of reference $[38,39]$, which verify the correctness of this model. Contrasting Figures 14(a) and 14(e), 15(a) and 15(e), it can be concluded that when the samples are small, the internal electromagnetic field and temperature distribution are similar between cubic and spherical samples. Contrasting Figures 14(b) and 14(f), or Figures 14(c) and 14(g), the electromagnetic field distribution of intermediate size samples is quite different between cubic and spherical samples. In spherical samples the focusing appears in the center, while in the cubic samples the focusing appears in the center as well as near the corners, which tend to be cyclical generation as the side length increases. Therefore in Figures 15(b) and 15(f), or Figures 15(c) and 15(g), hot spots in spherical and cubic samples are different in the aspect of position and number. Contrasting Figures 14(d) and 14(h), the electromagnetic field of spherical samples moves towards the outer surface direction and that of cubic samples is mostly focused on the corners, the corresponding temperature distribution in Figures 15(d) and 15(h) shows similar phenomena.

According to Figures 10-15, the samples can be divided into four groups. (1) Small volume samples, whose PAE and COV value are both low; besides, internal electromagnetic field focusing and hot spots are not obvious, as shown in Figures 14(a) and 14(e) and Figures 15(a) and 15(e), respectively. (2) Low intermediate volume samples, whose PAE values are low, while the COV values are high due to the concentrated internal hot spots, which are caused by the intense focusing effect of electromagnetic field, as shown in Figures 14(b) and 14(f) and Figures 15(b) and 15(f). (3) High intermediate volume samples, whose PAE values are high, while the COV values are low. The reasons that caused these phenomena are less concentrated hot spots and less intense electromagnetic focusing effect, as shown in Figures 14(c) and 14(g) and Figures 15(c) and 15(g). (4) Big volume samples, whose PAE values tend to be low or stable, and the COV values increase. The electromagnetic focusing and hot spots move to the outer surfaces and sharp corners, as shown in Figures 14(d) and 14(h) and Figures 15(d) and 15(h). In this section, samples in Study 1 Study 7 can be classified into the first group, those in Study 8 Study 25 can be classified into the second group, those in Study 26 Study 40 can be classified into the third group, and those in Study 41 Study 57 can be classified into the last group.

In summary, when the isometric volume spherical and cubic samples' sizes are small, the microwave power absorption capability of both shapes is similar, but the temperature distribution uniformity of cubic samples is worse than that of spherical samples. So on this condition, it is recommended to make samples sphere in microwave heating progress. In the case of intermediate size, the spherical samples' electromagnetic field focusing is in the center, while that of cubic ones locates both in center and on corners that result in multiple hot spots, so the PAE values of cubic samples are higher, and $\mathrm{COV}$ values of spherical samples are lower or comparable to the cubic ones. Thus, for the intermediate size samples, it is recommended to select the shape of the sample according to the demand for power absorbing capability or temperature uniformity. As for the big size samples, electromagnetic field distribution of spherical samples moves towards outer surface and that of cubic samples focuses on corners. The PAE values of cubic samples are higher than spherical ones, but the internal temperature uniformity gets worse and worse. Therefore, for the big size cubic samples are unpractical due to the worse temperature uniformity resulting from the focusing on corners.

3.2.2. Cylindrical, Cuboidal, and Ellipsoidal Sample. For cylindrical, cuboidal, and ellipsoidal samples, the same volume may have a variety of size combinations; namely, same volume samples may have different combinations of axial size and radial cross-sectional area. Therefore, in this section, the cylindrical, cuboidal, and ellipsoidal samples are of the same volume of $1.64 \times 10^{-5} \mathrm{~m}^{3}$; the samples' axial size is from $0.004 \mathrm{~m}$ to $0.163 \mathrm{~m}$. The radial cross sections of cylindrical and ellipsoidal samples are circular, whose diameters are from $0.016 \mathrm{~m}$ to $0.100 \mathrm{~m}$ and from $0.0196 \mathrm{~m}$ to $0.1225 \mathrm{~m}$, respectively, while the cross section of cuboidal sample is square, whose side length is from $0.0143 \mathrm{~m}$ to $0.0886 \mathrm{~m}$.

As shown in Figure 16, cylindrical, cuboidal, and ellipsoidal samples of the same volume were placed in three directions. The correlation results can be obtained by contrasting 


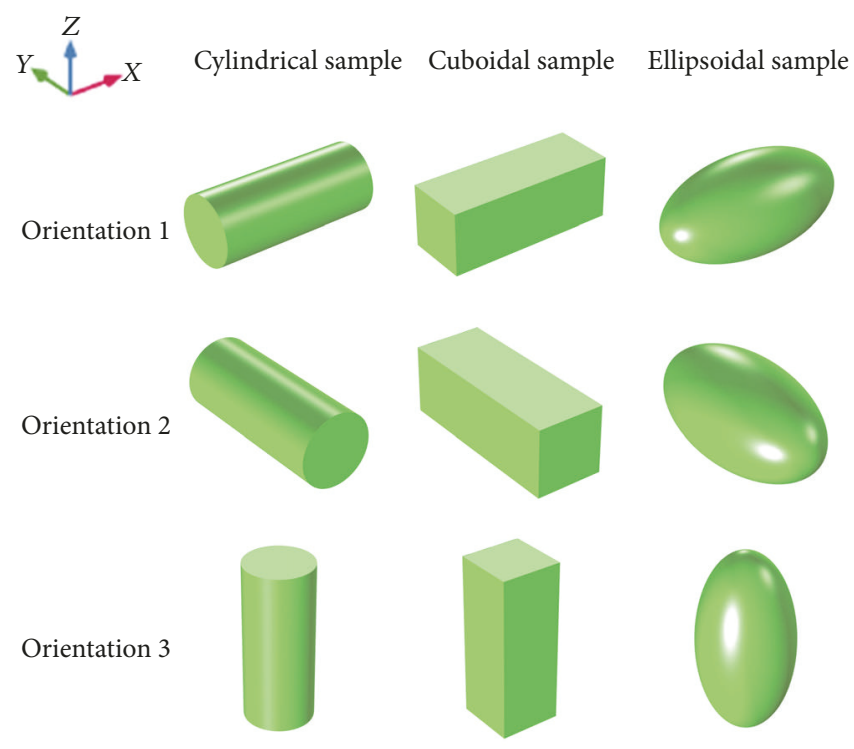

FIGURE 16: Sample diagram.

the different samples in same directions (horizontal) as well as the same sample in different directions (vertical), as discussed in this section and Section 3.3, respectively.

The thickness of samples can be described by wave number $N_{w}$ and penetration number $N_{p}$ defined as (10) in 2D problems [37]:

$$
\begin{aligned}
& N_{w}=\frac{L}{\lambda_{m}} \\
& N_{p}=\frac{L}{D_{p}},
\end{aligned}
$$

where $L$ is the characteristic dimension, and for circular cross section samples $L$ is the diameter, and for square cross section samples $L$ is the side length. In addition, $\lambda_{m}$ and $D_{p}$ are the wavelength and penetration depth of microwave within the food material, which can be obtained from the following equations [37]:

$$
\begin{aligned}
& \lambda_{m}=\frac{c \sqrt{2}}{f\left[\sqrt{\left(\varepsilon^{\prime}\right)^{2}+\left(\varepsilon^{\prime \prime}\right)^{2}}+\varepsilon^{\prime}\right]^{1 / 2}} \\
& D_{p}=\frac{c}{\sqrt{2} \pi f\left[\sqrt{\left(\varepsilon^{\prime}\right)^{2}+\left(\varepsilon^{\prime \prime}\right)^{2}}-\varepsilon^{\prime}\right]^{1 / 2}} .
\end{aligned}
$$

Thus, The wavelength and penetration depth within the potato samples of this section are $0.016 \mathrm{~m}$ and $0.023 \mathrm{~m}$, respectively.

In the 2D study in [37], different shape food samples could be classified as thin $\left(N_{w} \leq 0.1\right)$, intermediate $\left(N_{w}>0.1\right.$, $\left.N_{p}<3\right)$, and thick $\left(N_{p} \geq 3\right)$ size according to samples' wave number and penetration number on the cross-section plane. Similarly, in the 3D study of this section, potato samples can be classified on the $X Y$-plane, $Y Z$-plane, and $Z X$-plane in the same way. The wave numbers of three shapes samples on axial direction section are from 0.261 to 10.186 , and the penetration numbers are from 0.181 to 7.086. The wave numbers on cylindrical, cuboidal, and ellipsoidal samples' radial middle cross section are, respectively, from 1 to 6.250 , from 0.886 to 5.539 , and from 1.225 to 7.655 . The penetration numbers of them are, respectively, from 0.696 to 4.348 , from 0.617 to 3.853 , and from 0.852 to 5.352 .

For the thin size samples the temperature distribution uniformity is invariant of the shape [37]. Therefore, in this section, all the samples locate in the regions of intermediate and thick size. Besides, according to the radial and axial wave number and penetration number, the samples can be further divided into three categories, namely, (1) Group 1: radial thick-axial medium samples, whose radial penetration number is $N_{p}^{r}>3$ and the axial wave numbers and penetration numbers are $N_{w}^{a}>0.1$ and $N_{p}^{a}<3$; (2) Group 2: radial medium-axial medium samples, whose wave numbers and penetration numbers in radial and axial directions are all $N_{w}>0.1$ and $N_{p}<3$; (3) Group 3: radial mediumaxial thick samples, whose radial penetration numbers are $N_{w}^{r}>0.1$ and $N_{p}^{r}<3$, and axial penetration numbers are $N_{p}^{a}>3$. Intuitively, samples of Group 1 tend to be more flat, while those belonging to the Group 3 are more slender.

When the samples are placed in Orientation 1, the electromagnetic field distribution on radial middle cross section ( $Y Z$ section) demonstrates unique characteristics of different samples; some typical results are shown in Figure 17. Images in Figure 17(a) belong to Group 1; the electromagnetic field focusing almost locates near the circular's surface and square's surface and corners. Images in Figures 17(b) and 17(c) show the inward focusing trend of samples in Group 2. Images in Figure 17(d) exhibit the electromagnetic focusing in the center of samples in Group 3. The above results are consistent with 2 D results of thick turnip samples in [37]. 

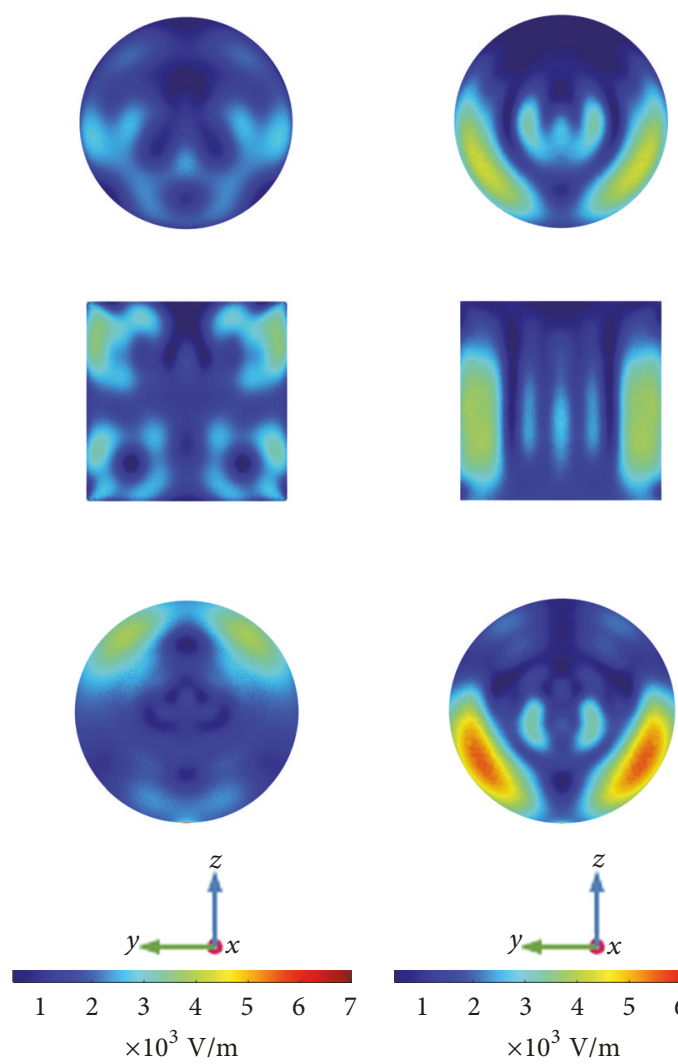

(a) $H=0.004 \mathrm{~m}$

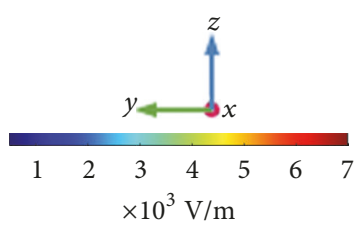

(b) $H=0.010 \mathrm{~m}$
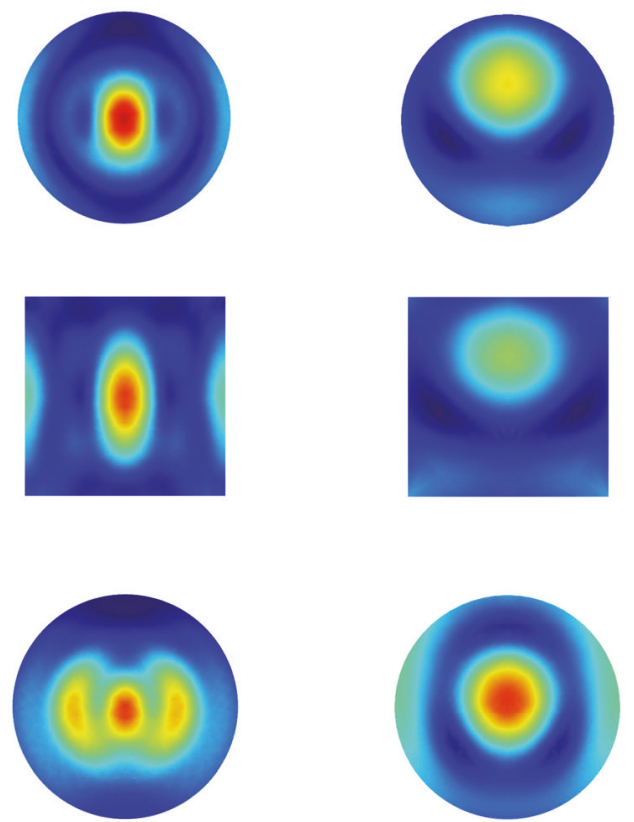

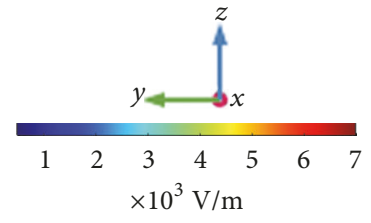

(c) $H=0.032 \mathrm{~m}$

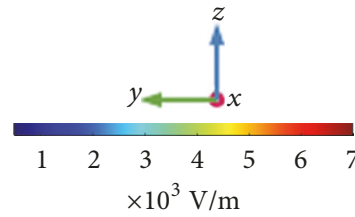

(d) $H=0.163 \mathrm{~m}$

FIGURE 17: Electromagnetic field distribution on $Y Z$ radial middle cross section in Orientation 1.

Besides, the corresponding axial cross sections ( $X Y$ section and ZX section) of these samples are shown in Figures 18 and 19. Considering the geometric optical properties of microwave, electromagnetic field in the cavity consists of the superposition of the original electromagnetic wave with that reflected by walls and samples' surfaces. Besides, the electromagnetic field in the foodstuff originates from the electromagnetic wave refracted from its surfaces [36]. In this section, the refraction surfaces of samples include two end surfaces and a cambered surface (as for cuboidal samples the cambered surfaces are those except for the two end surfaces).

Therefore, it is clear that when the size of axis is less than penetration depth and wavelength, the focusing is mainly caused by the microwave refracted from the cambered surface as shown in Figures 18(a) and 19(a).

As shown in Figures 18(b) and 19(b), sample's radial size is bigger than penetration depth and the axial size is comparable to wavelength, so the focusing appears separately near cambered surface as well as in the internal space due to the microwave refracted from cambered surface and end surfaces, respectively. For samples whose radial size and axial size are both close to the penetration depth, the microwave refracted from the cambered surface and the two end surfaces all play important roles in the internal electromagnetic wave propagation, and the results of intensive superposition are shown in Figures 18(c) and 19(c).
In Figures 18(d) and 19(d), sample's radial sizes are comparable to the wavelength, while the axial sizes are bigger than penetration depth. Thus, the electromagnetic focusing along the axis is mainly caused by the microwave refracted from the cambered surface, and only within the penetration depth region near end surfaces may microwave refraction from two end surfaces have an effect on the microwave superposition.

In Figures 17-19, we can find that the electromagnetic field distribution of same axial size samples presents similar tendency and local differences, and from the electromagnetic field distribution images it is not hard to obtain the distribution of temperature. However, from the intuitional visual images it is difficult to determine the optimal samples of high power absorption and uniform temperature distribution. Therefore, parameter of power absorption efficiency (PAE) is used to measure the samples' microwave power absorbing capability, and a higher PAE value is desired. Besides, coefficient of variation (COV) of samples' temperature is used for evaluating the internal temperature of samples, and the low COV value demonstrates a better temperature uniformity. Moreover, optimal sample should own high power absorption efficiency and low temperature $\mathrm{COV}$ value; thus the ratio of power absorption efficiency to temperature $\mathrm{COV}$ value (named comprehensive evaluation coefficient, CEC) can measure the samples' usability at a certain condition, and a 


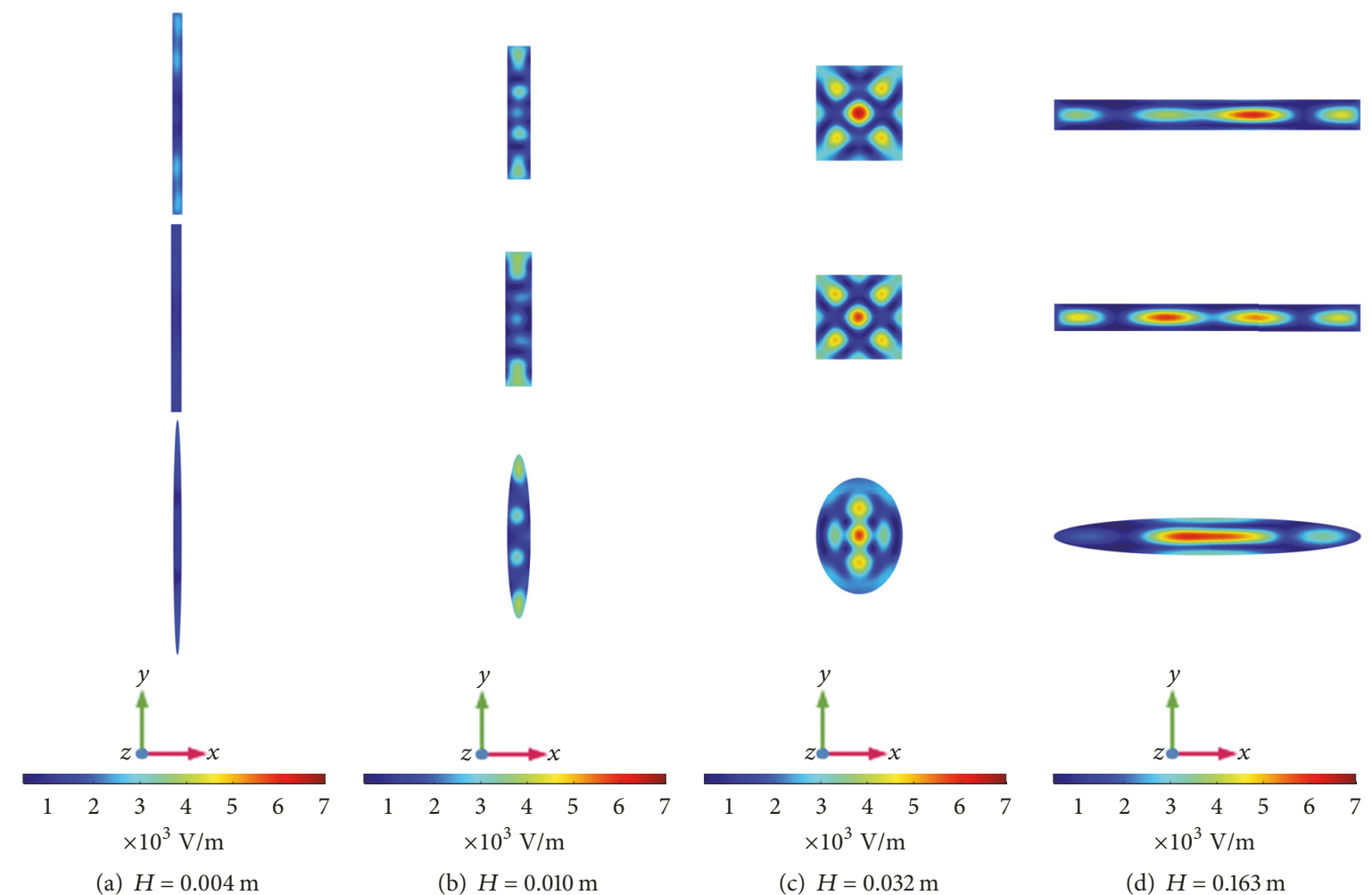

FIGURE 18: Electromagnetic field distribution on $X Y$ axial middle cross section in Orientation 1.

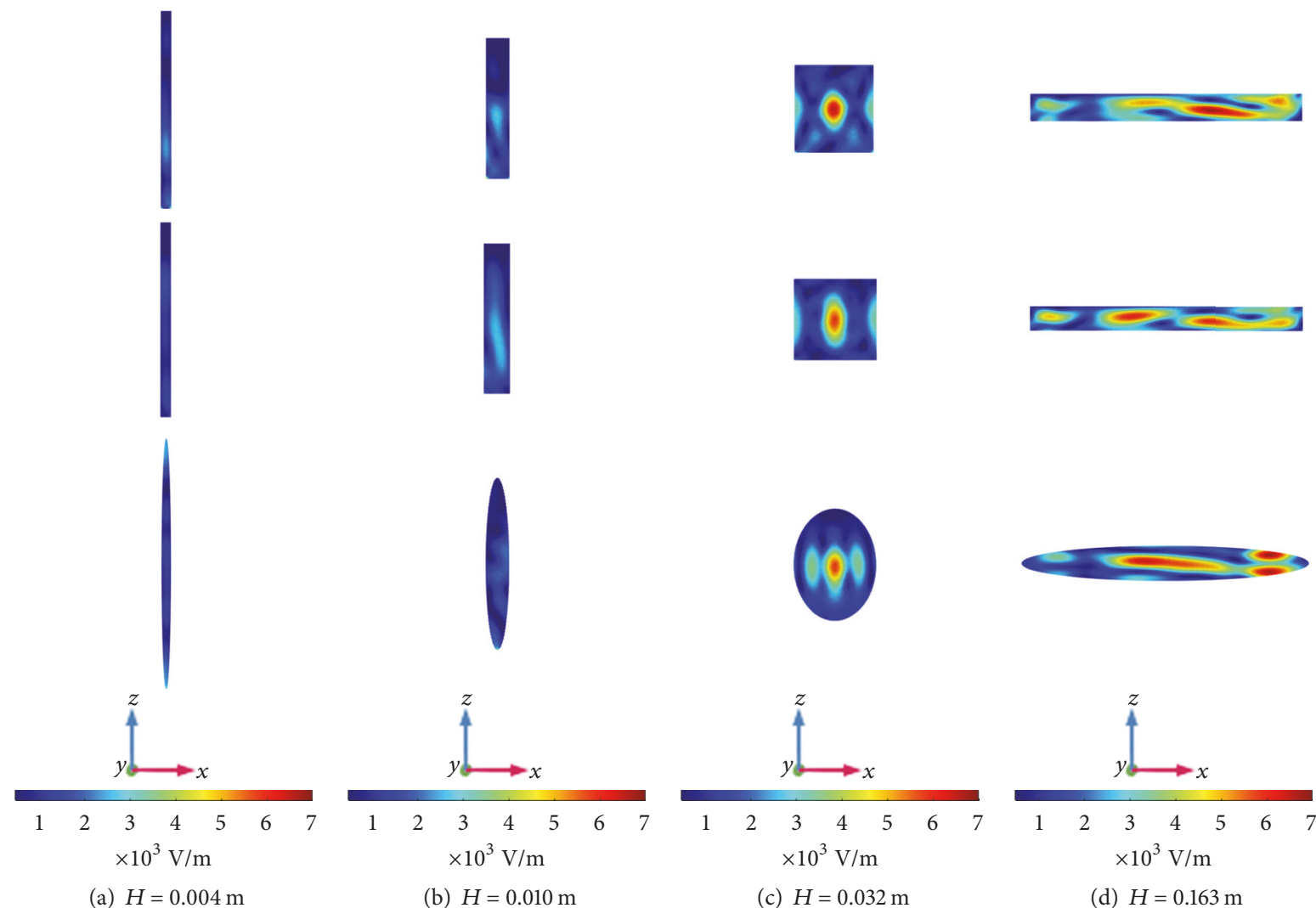

FIGURE 19: Electromagnetic field distribution on $Z X$ axial middle cross section in Orientation 1. 


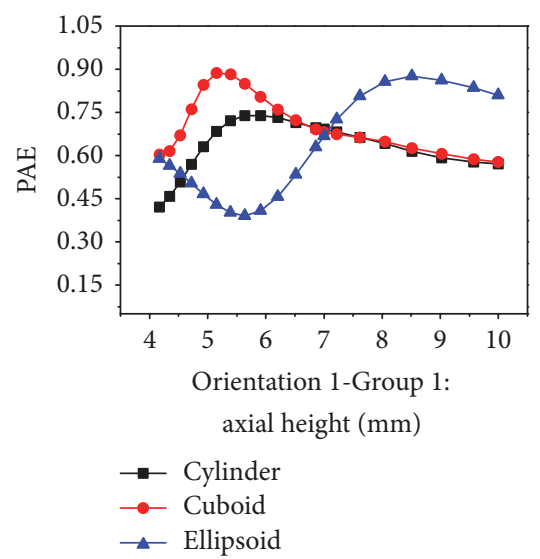

(a)

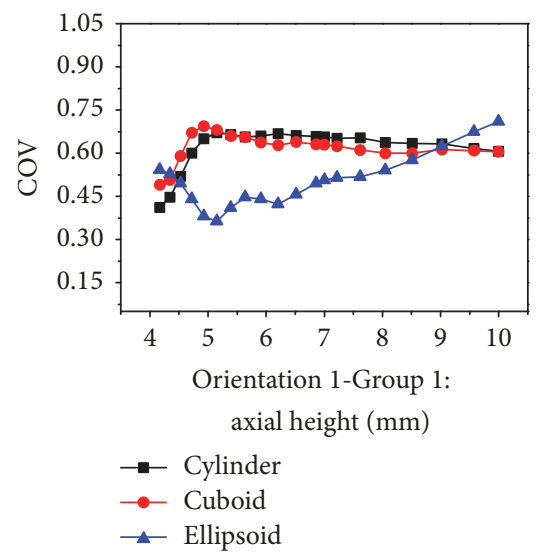

(d)

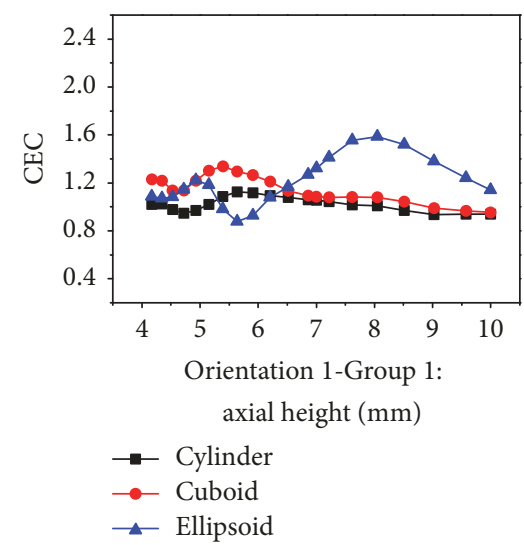

(g)

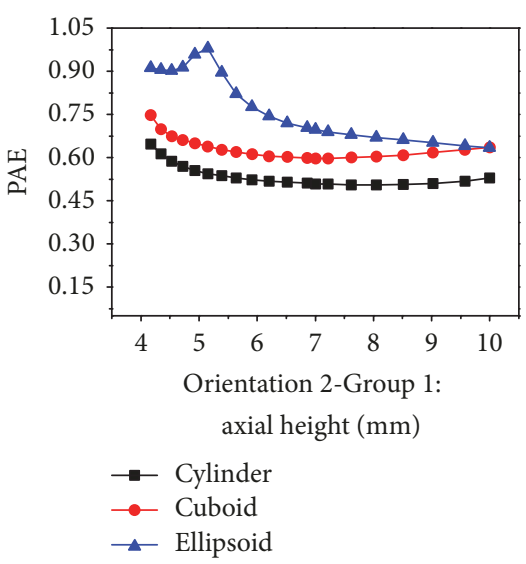

(b)

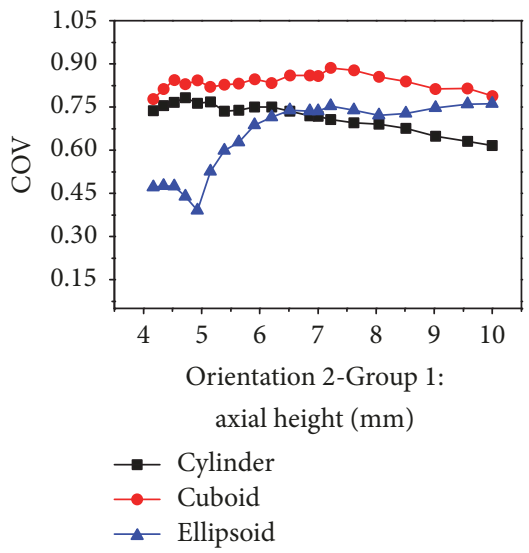

(e)

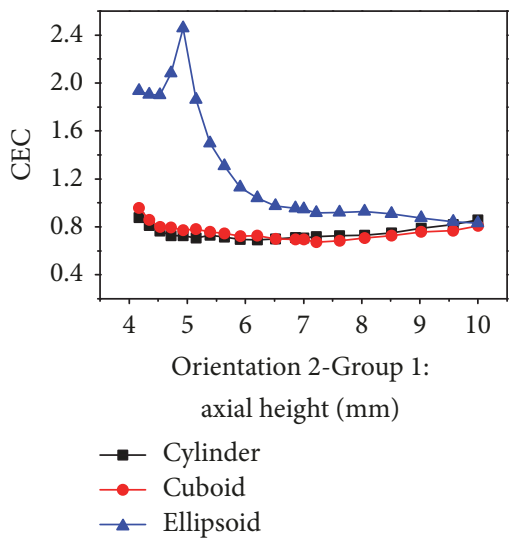

(h)

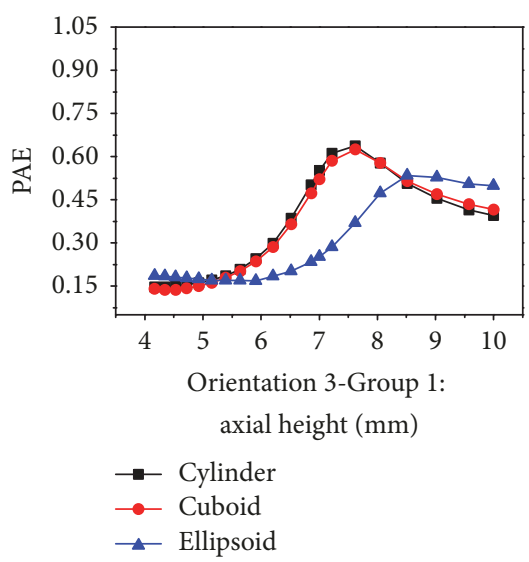

(c)

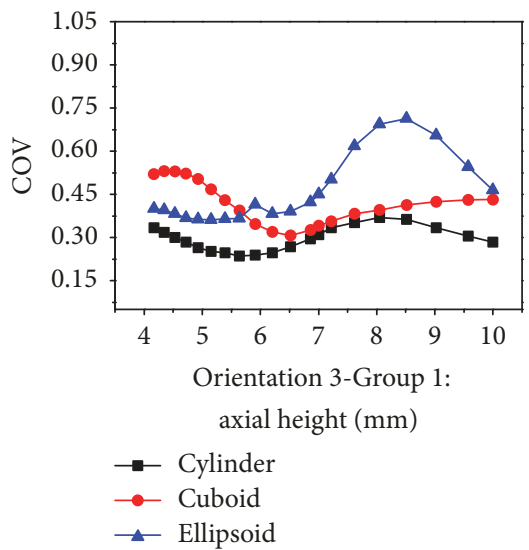

(f)

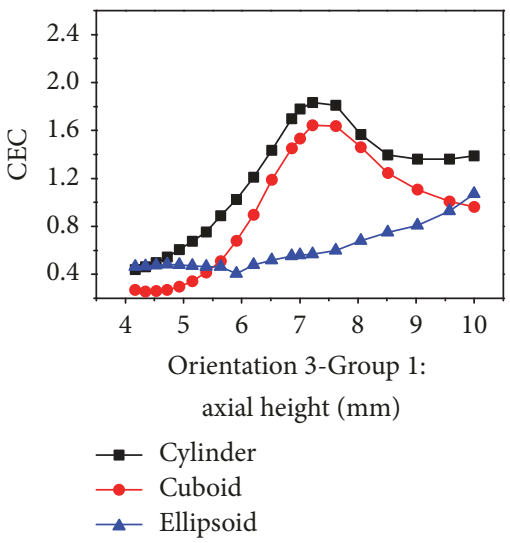

(i)

FIGURE 20: Different samples in the same orientations, Group 1.

higher CEC value presents a sample with a high PAE and/or low COV, which could be chosen according to specific needs of microwave process.

Curves in Figures 20(a)-20(c) (similarly in Figures 21(a)-21(c) and Figures 22(a)-22(c)) give the PAE values of these three shape samples in different orientations. Besides, when the internal hot spots reach the phase transition temperature, curves in Figures 20(d)-20(f) (similarly in Figures 21(d)-21(f) and Figures 22(d)-22(f)) demonstrate the temperature COV values of three shape samples in different orientations. Figures 20(g)-20(i) (similarly Figures 21(g)-21(i) and Figures 22(g)-22(i)) give the corresponding CEC curves of the three shape samples in different orientations.

In Figures 20(a), 20(d), and 20(g), the figures suggest that, in Orientation 1, cylindrical, cuboidal, and ellipsoidal samples' PAE values have maximal values which are $0.74,0.89$, and 0.88 , and the corresponding COV values are also located 


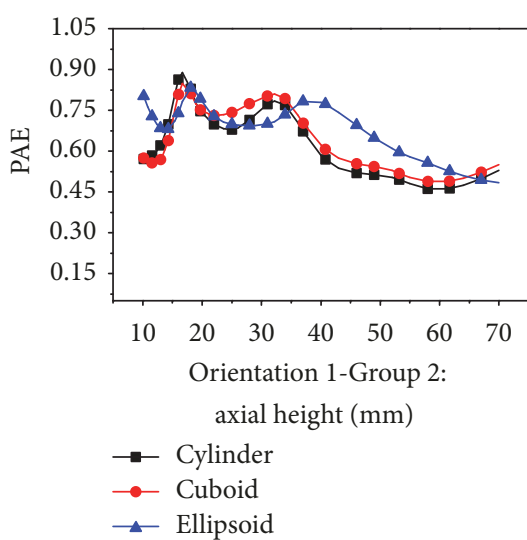

(a)

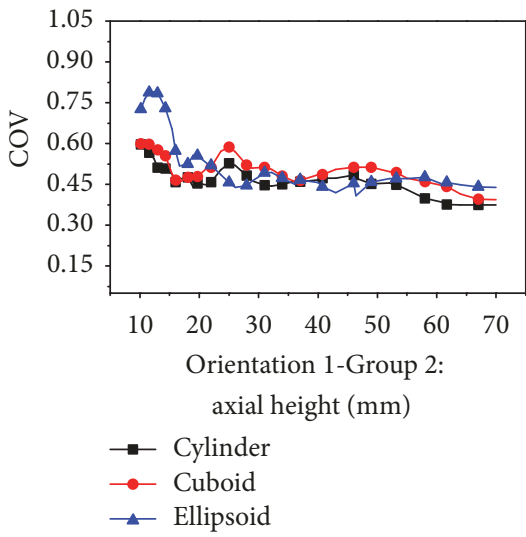

(d)

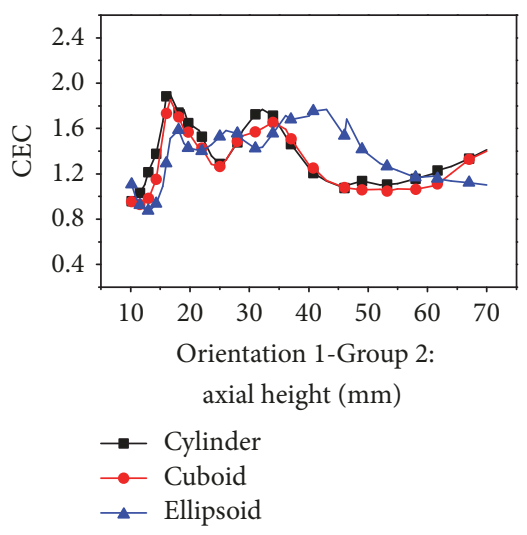

(g)

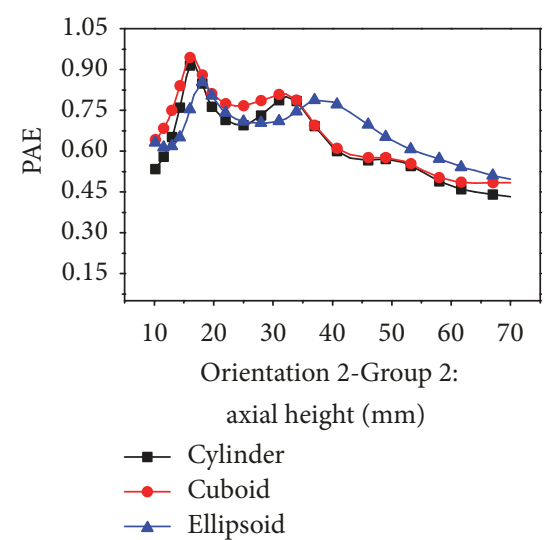

(b)

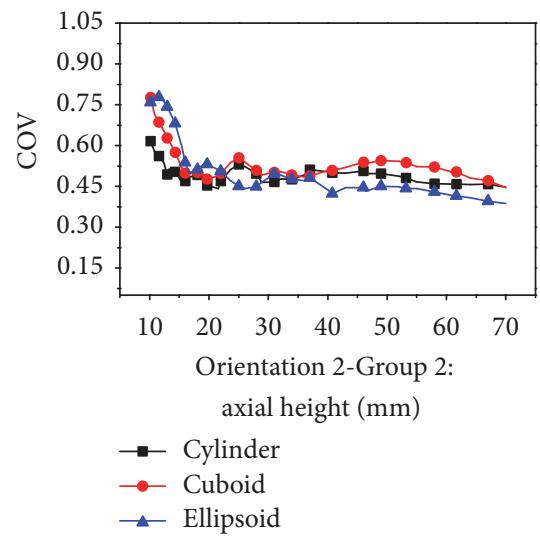

(e)

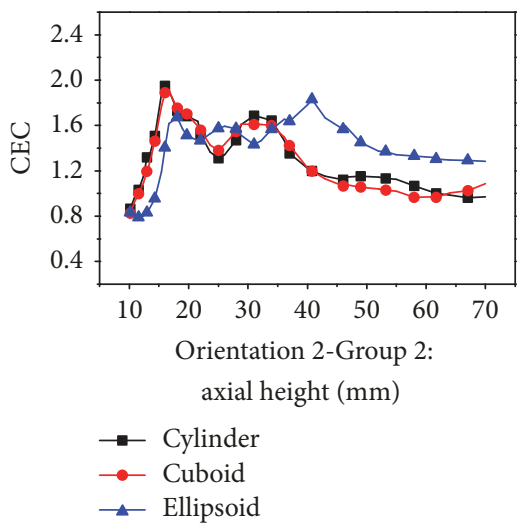

(h)

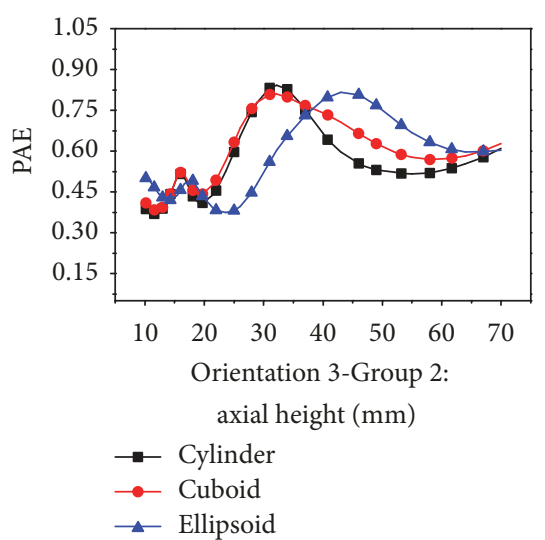

(c)

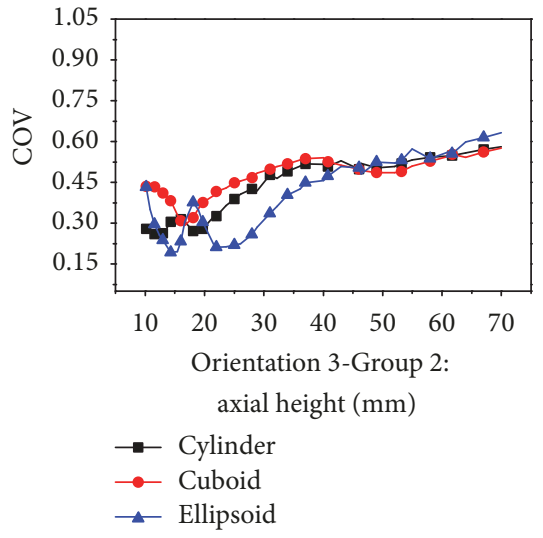

(f)

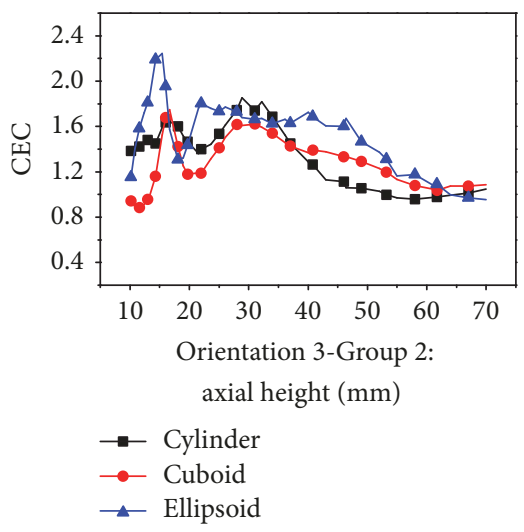

(i)

FIGURE 21: Different samples in the same orientations, Group 2.

near the maximal values except ellipsoidal sample. Thus from Figure $20(\mathrm{~g})$ it can be concluded that when samples of Group 1 are placed in Orientation 1, sample of ellipsoidal shape is better than cylindrical and cuboidal ones due to relatively high PAE and low temperature COV. Similarly, in Figures 20(b), 20(e), and 20(h), when samples are placed in Orientation 2, ellipsoidal shape is also the best choice. However when samples are placed in Orientation 3 as shown in Figures 20(c), 20(f), and 20(i), cylindrical shape samples are better than the other two shapes for the same reason.
PAE, COV, and CEC value curves of different samples in Group 2 are shown in Figure 21. From the curves in Figures 21(g)-21(i), it can be concluded that when samples' axial sizes are lower than $0.040 \mathrm{~m}$, samples' CEC values are all comparable. The reason causing this phenomenon is that the radial and axial sizes of samples are all comparable to the penetration depth or wavelength, and thus intensively electromagnetic field focusing would happen to enhance the heating effect. Among this range, samples' shape effect can be ignored, and all these three shape samples can obtain good 


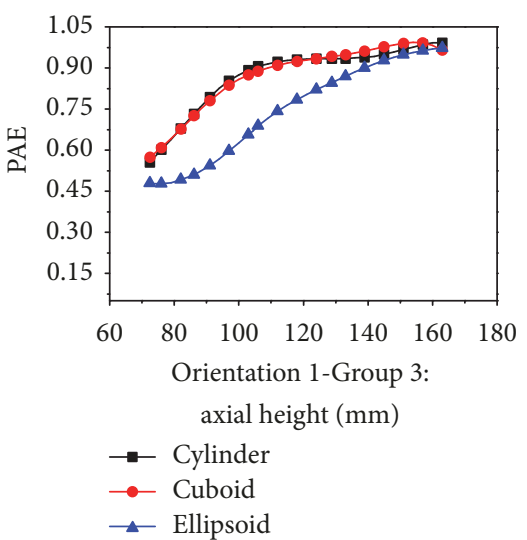

(a)

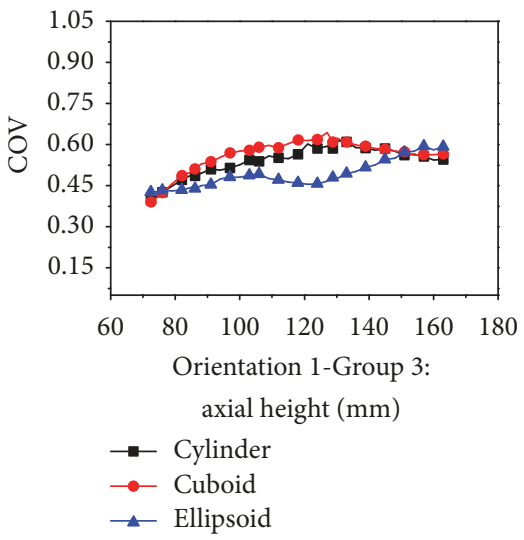

(d)

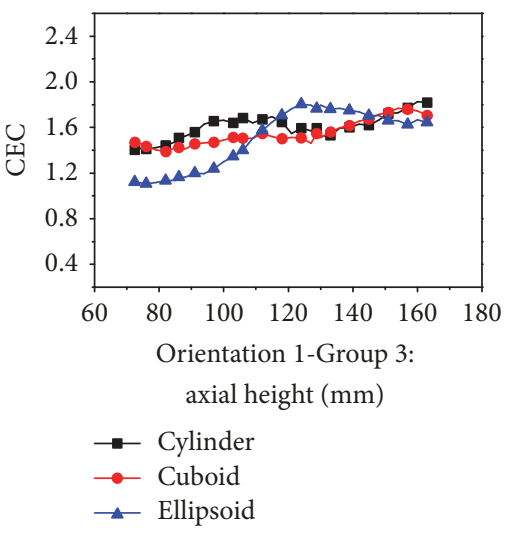

(g)

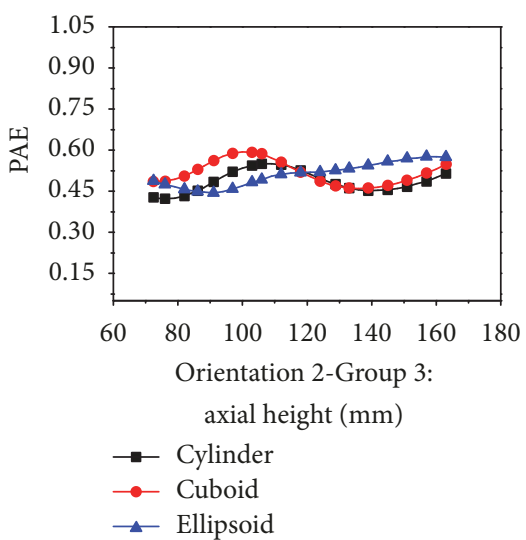

(b)

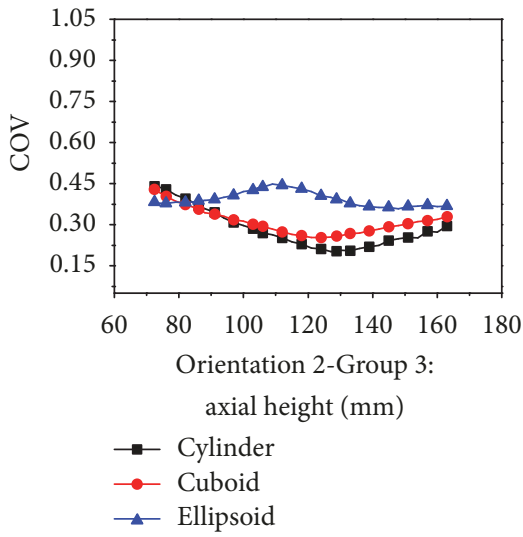

(e)

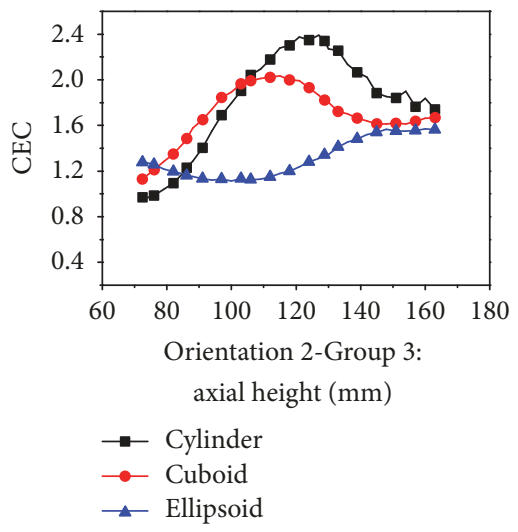

(h)

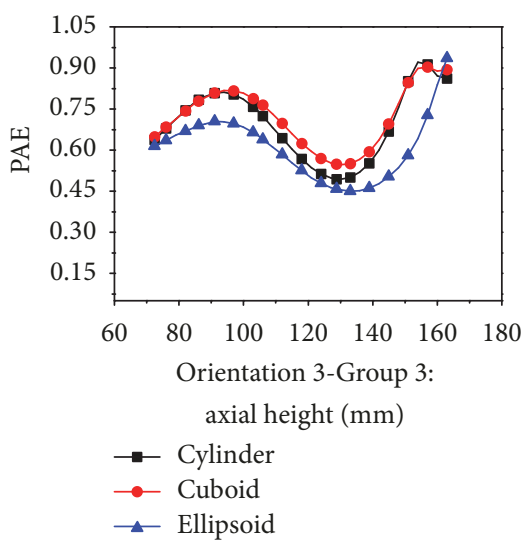

(c)

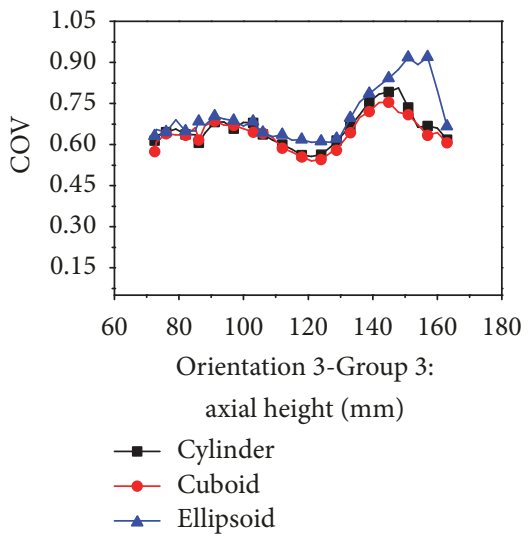

(f)

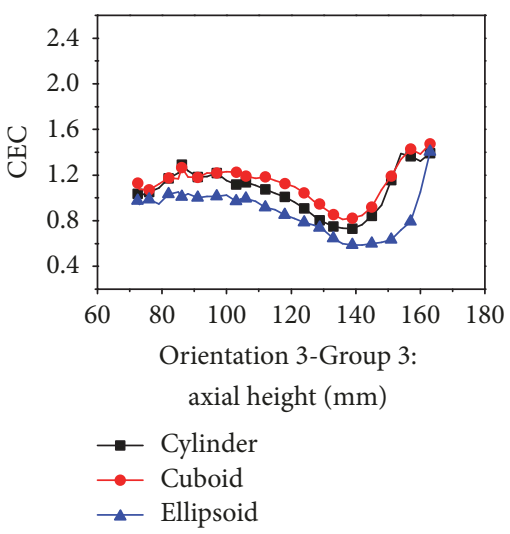

(i)

FIGURE 22: Different samples in the same orientations, Group 3.

heating results. Beyond this range ellipsoidal shape would be better because of the higher PAE and/or lower COV.

PAE, COV, and CEC of samples in Group 3 are shown in Figure 22. When placed in Orientation 1, cylindrical samples are the first choice due to the higher PEA and relatively lower COV as shown in Figures 22(a), 22(d), and 22(g). Cylindrical and cuboidal shapes are better than ellipsoidal shape when placed in Orientation 2. Besides, if temperature uniformity is more decisive, cylindrical shape is the best. When placed in Orientation 3, cuboidal shape should be firstly selected.
Taking all samples in the three groups into consideration, in Figures 23-25, every curve is divided into three parts: the left unmarked part separately indicates the PAE, COV, and CEC values of the samples belonging to Group 1 and the middle part and right part represent Group 2 and Group 3 as marked in figures.

In Figure 23(a), it can be seen that, for all samples whose axial sizes are in the range of $0.004 \mathrm{~m}$ to $0.163 \mathrm{~m}$, cylindrical and cuboidal samples' PAE values are almost equal to each other in Orientation 1. The PAE value curve of ellipsoidal 


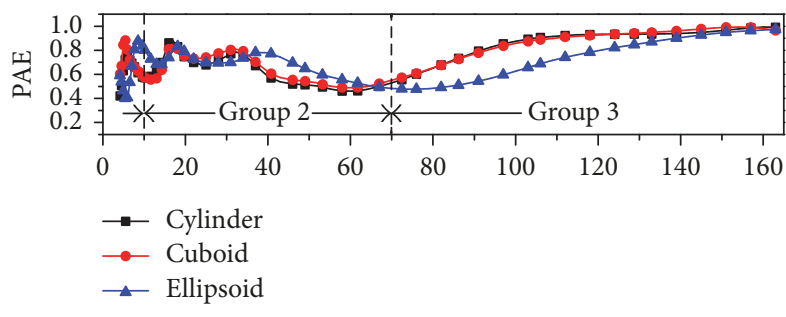

(a) Orientation 1: axial height (mm)

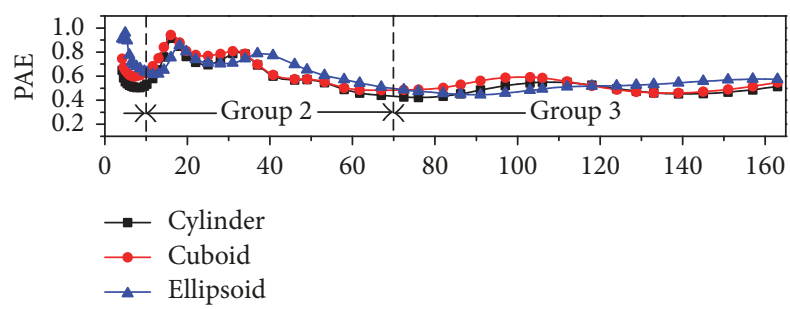

(b) Orientation 2: axial height (mm)

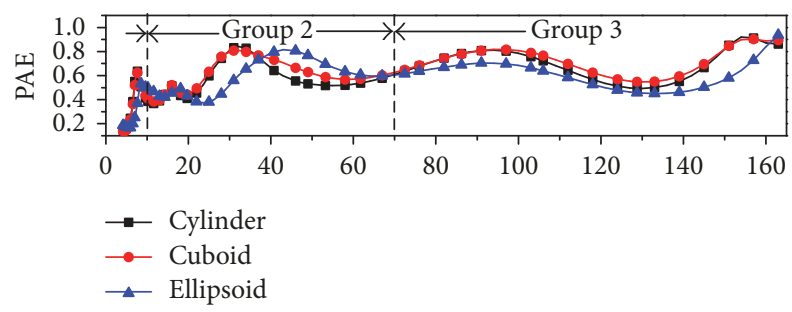

(c) Orientation 3: axial height (mm)

FIgure 23: PAE of different shapes.

samples is interlaced with those of the other two shapes. This phenomenon is caused by the equal radial cross section areas of cylindrical and cuboidal samples whose axial sizes are equal and of cylindrical and ellipsoidal samples whose axial sizes are not equal. Similar phenomena are also shown in Figures 23(b) and 23(c).

In Figures 24(a)-24(c), when samples' axial sizes are lower than $0.020 \mathrm{~m}$, the temperature COV curves show intense volatility. As the axial sizes of samples are bigger than $0.020 \mathrm{~m}$, COV curves in Figure 24(a) are relatively flat, those in Figure 24(b) show downward tendency, and those in Figure 24(c) show upward tendency. Therefore, when samples' axial sizes increase from Group 1 to Group 3, samples' temperature uniformity becomes stable in Orientation 1, gets better in Orientation 2, and gets worse in Orientation 3. It suggests that different shape samples in the same orientation have the same temperature uniformity variation tendency.

In Figures 25(a)-25(c), it can be concluded that in every sample group there exist extreme values, which means there are optimal sample shape in a certain range as discussed previously. In Figures 25(a) and 25(b), some of the CEC values in Group 3 are bigger than those in Group 2, but the causes of the maximal values of these two groups are different. So when selecting sample shapes, only considering CEC value is not enough; PAE and COV values should be considered as well. As discussed previously, the optimal shape in Group 2 is selected due to the two optimal values of PAE and COV,

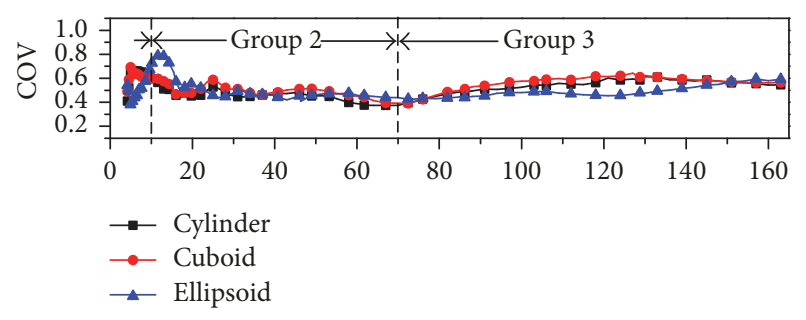

(a) Orientation 1: axial height ( $\mathrm{mm})$

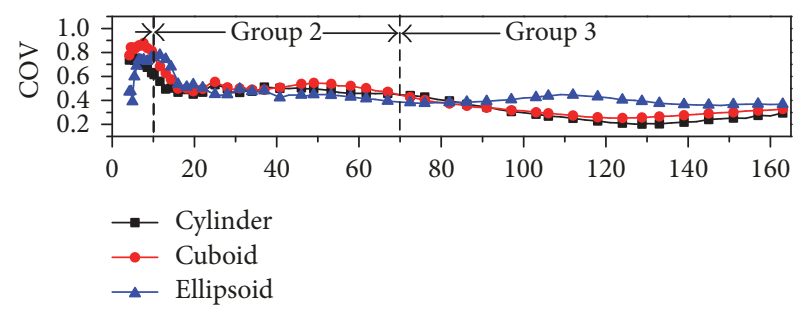

(b) Orientation 2: axial height (mm)

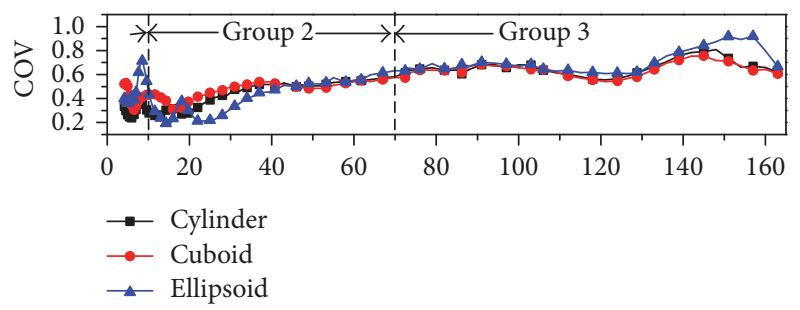

(c) Orientation 3: axial height (mm)

Figure 24: COV of different shapes.

namely, higher PAE and lower COV. However, in Group 3, due to the fact that the optimal values of PAE and COV cannot be obtained synchronously, optimal shape should be selected according to specific need of temperature uniformity or microwave power absorption capability.

To sum up, in this section microwave heating properties of samples of different shapes placed in the same orientation were elaborated by means of intuitive figures and quantificational curves. Different shape samples were classified into three categories according to the radial and axial wave number and penetration number. Samples belonging to different groups demonstrate different electromagnetic field and temperature distribution. PAE, COV, and CEC curves are used to comprehensively evaluate the usability of a sample. PAE, COV, and CEC curves of different shape samples in same orientation have similar variation tendency. For samples belonging to Group 1, ellipsoidal shape sample is the optimal shape when placed in Orientation 1 and Orientation 2 , and when the sample is placed in Orientation 3, cylinder is the best shape. For samples belonging to Group 2, when samples' axial sizes are lower than $0.040 \mathrm{~m}$, the effect of sample shape is small enough to be negligible, and beyond this range ellipsoidal shape would be a better choice. For samples in Group 3, cylindrical samples are better when placed in Orientation 1 and Orientation 2, and when in Orientation 3, cuboid would be a better shape. 


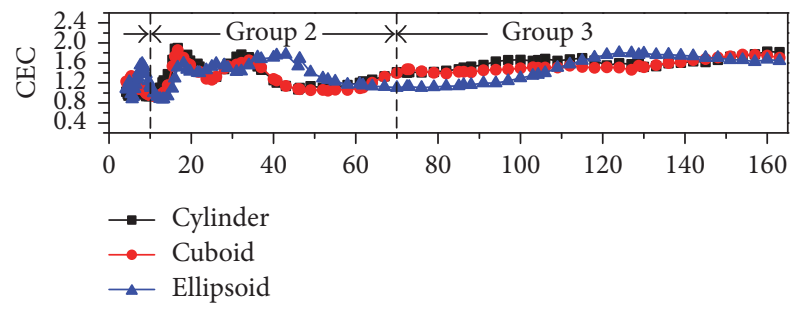

(a) Orientation 1: axial height ( $\mathrm{mm})$

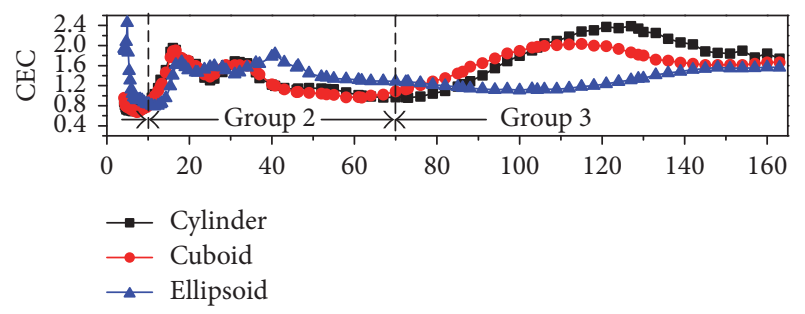

(b) Orientation 2: axial height $(\mathrm{mm})$

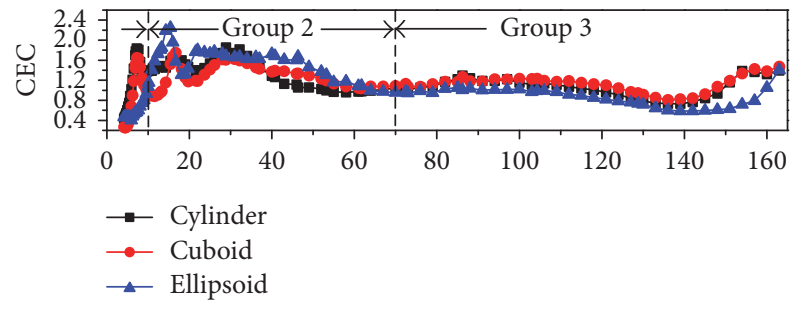

(c) Orientation 3: axial height $(\mathrm{mm})$

FIGURE 25: CEC of different shapes.

3.3. Position Orientation Effect. In Section 3.2.2, microwave heating properties of samples of different shapes placed in the same orientation have been discussed. During the studies, it is clear that when samples' shapes changed, the quantificational curve differed significantly. Meanwhile, differences between samples of the same shape in different orientations were also obvious. Therefore, in this section the microwave heating properties of the same samples placed in three orientations (shown in Figure 16) are discussed as follows.

PAE, COV, and CEC values of samples in Group 1 placed in different orientations are shown in Figure 26. From Figures 26(a), 26(d), and 26(g), it can be seen that PAE values of cylindrical samples placed in Orientation 1 are bigger than other orientations, but the corresponding temperature COV values of cylindrical samples placed in Orientation 3 are better than the others. Besides, the CEC values of cylindrical samples placed in Orientation 3 are better than those placed in Orientation 1. Therefore, if the microwave heating process prefers a high power absorption capability, cylindrical samples in Group 1 should be placed in Orientation 1. If temperature uniformity is a more decisive factor, cylindrical samples in Group 1 should be placed in Orientation 3. The same conclusion can be drawn for the cuboidal samples in Group 1. For ellipsoidal samples, when sample axial size is small enough, Orientation 2 would be a good choice due to a higher PAE and lower COV. Otherwise, Orientation 1 is better for the same reasons.
PAE, COV, and CEC values of samples in Group 2 placed in different orientations are shown in Figure 27. From Figures 27(a)-27(f), it can be seen that when samples' axial sizes are lower than $0.040 \mathrm{~m}$, the PAE and COV values of samples placed in Orientation 3 are lower than the other two orientations. When the axial sizes are bigger than $0.040 \mathrm{~m}$, those values of Orientation 3 are larger than the others. Thus, in Figures 27(g)-27(i), the CEC values of different orientations are close to each other. Therefore, when selecting the orientations of samples in Group 2, users should firstly confirm whether the microwave absorption efficiency or the temperature uniformity is the decisive factor. If high PAE is more important, samples should be placed in Orientation 1 and Orientation 2 for those whose axial sizes are lower than $0.040 \mathrm{~m}$ and in Orientation 3 for those whose axial sizes are bigger than $0.040 \mathrm{~m}$. Otherwise, the lower COV values would be the dominant factor. As the conclusion drawn in former section, the effect of sample shape in Group 2 is less important than that of orientation.

PAE, COV, and CEC values of samples in Group 3 placed in different orientations are shown in Figure 28. It is clear that samples' PAE values of Orientation 1 are the highest and those of Orientation 3 are the lowest as shown in Figures 28(a)-28(c). Besides, the COV values of Orientation 2 are the best and those of Orientation 3 are the worst as shown in Figures 28(d)-28(f). Thus, from the CEC value shown in Figures 28(g)-28(i), the first conclusion that can be drawn is for the samples in Group 3: Orientation 3 is the worst choice to place the samples. Secondly, taking the PAE and COV values into consideration, if the microwave process needs higher PAE, Orientation 1 is the best choice. If temperature COV value is the dominant factor, it is better to place the samples in Orientation 2 though the PAE values are only around 0.45 on this condition.

PAE, COV, and CEC values of samples in three groups are shown in Figures 29-31, respectively. In these images, positions of Group 2 and Group 3 have been marked and the unmarked part is belonging to the Group 1.

In Figure 29, it can be seen that when samples' axial sizes are lower than $0.040 \mathrm{~m}$, the PAE values of samples placed in Orientation 3 are lower than those of samples placed in the other two orientations. When the axial sizes are bigger than $0.040 \mathrm{~m}$, the PAE curves of Orientation 1 tend to a fixed value of about 0.9 , curves of Orientation 2 fluctuate around $0.52,0.55$, and 0.56 for cylindrical, cuboidal, and ellipsoidal samples, respectively, and curves of Orientation 3 fluctuate around $0.67,0.70$, and 0.64 for cylindrical, cuboidal, and ellipsoidal samples, respectively. Obviously, from the perspective of microwave power absorption capability of samples, Orientation 1 is the best choice for all the three groups of samples.

In Figure 30, when samples' axial sizes are lower than $0.040 \mathrm{~m}$, the temperature COV values of samples placed in Orientation 3 are the lowest among three orientations; namely, samples could obtain the best temperature uniformity on this condition. However, when the axial sizes of samples are bigger than $0.040 \mathrm{~m}$, the temperature COV values of Orientation 3 get worse which are of average values of about $0.61,0.60$, and 0.65 separately of cylindrical, 


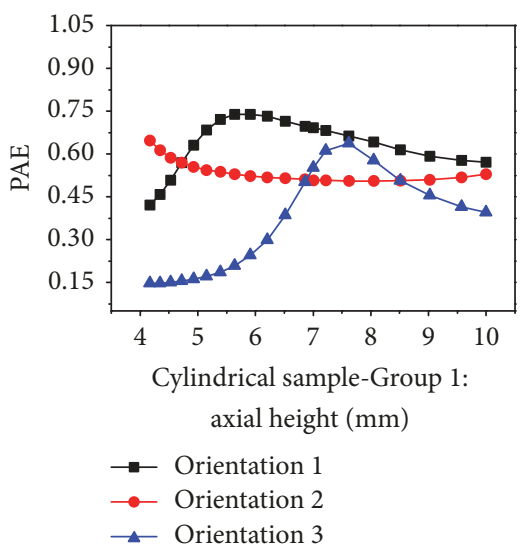

(a)

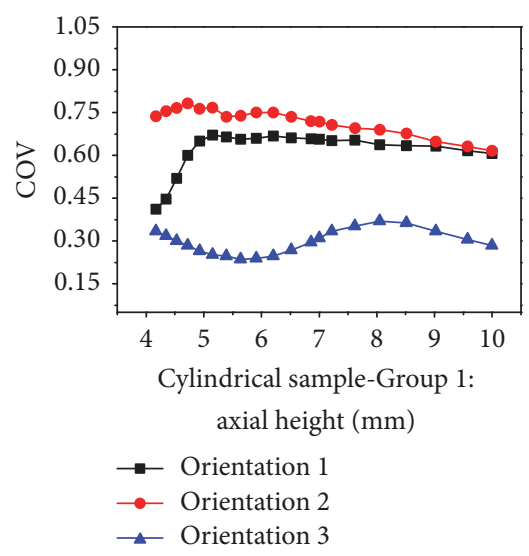

(d)

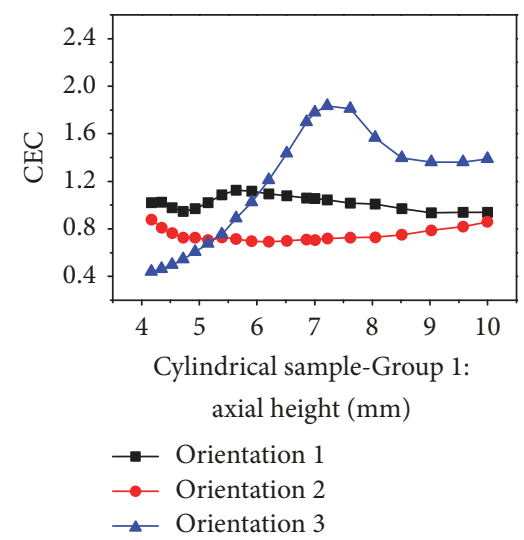

(g)

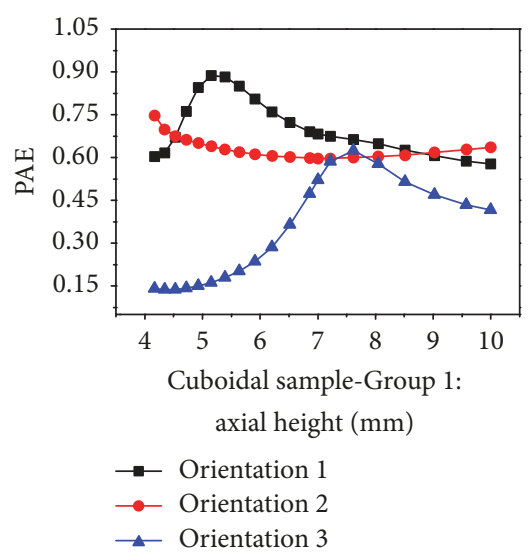

(b)

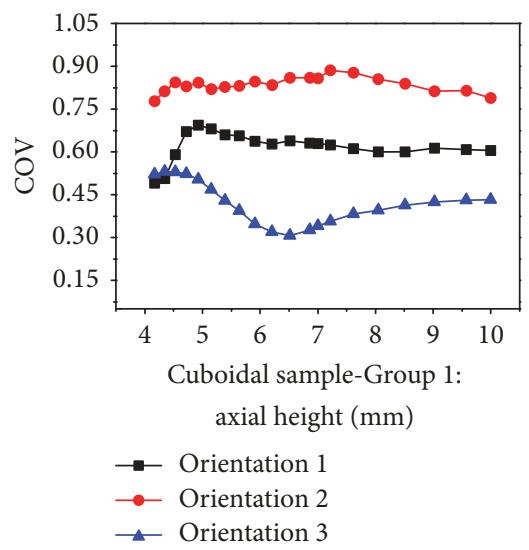

(e)

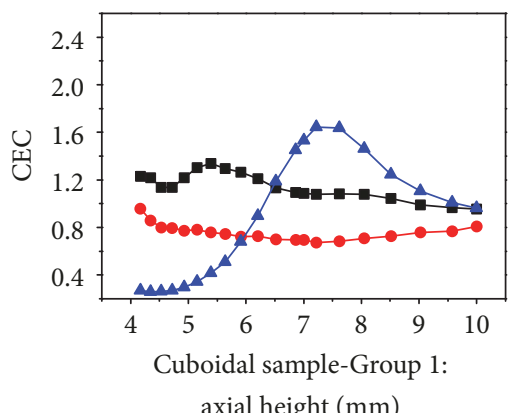

axial height $(\mathrm{mm})$

- Orientation 1
$\rightarrow$ Orientation 2
- Orientation 3

(h)

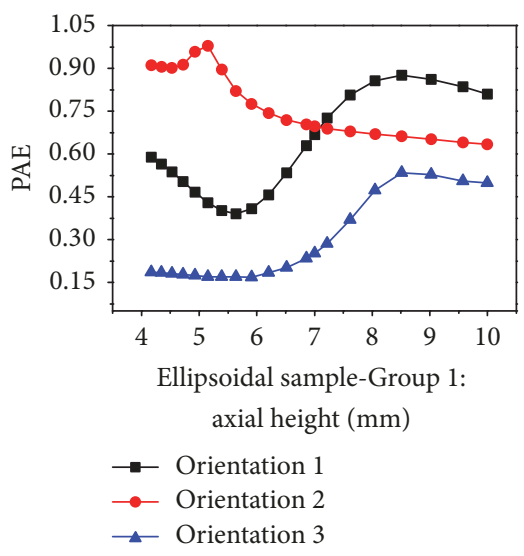

(c)

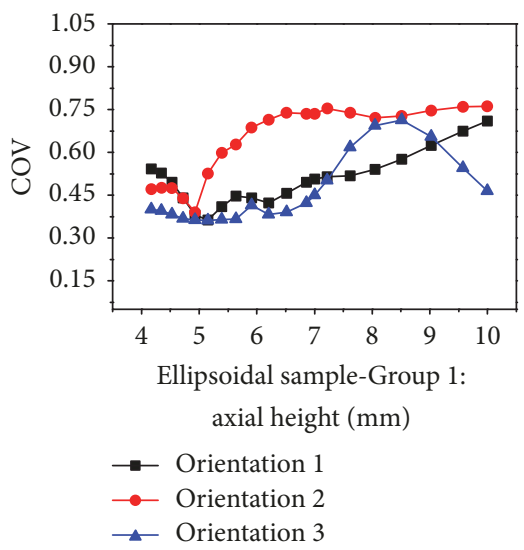

(f)

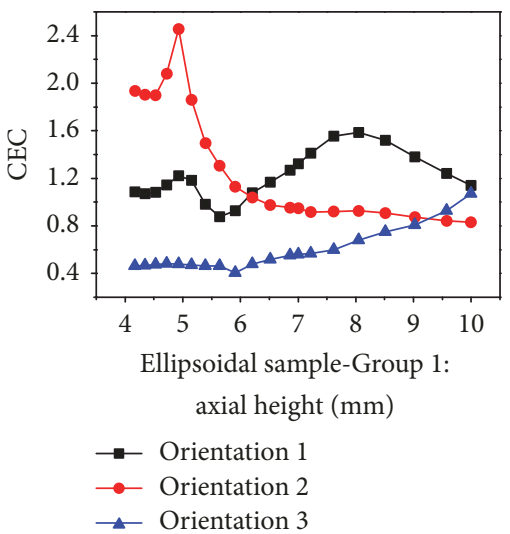

(i)

FIGURE 26: Same samples in different orientations, Group 1.

cuboidal, and ellipsoidal samples. Those of Orientation 2 get better which are of average values about $0.34,0.37$, and 0.40 separately of cylindrical, cuboidal, and ellipsoidal samples. And values of Orientation 1 keep stable average values of $0.50,0.53$, and 0.48 , respectively. Therefore, if a microwave process has a higher requirement for temperature uniformity, Orientation 2 is the best choice for samples whose axial sizes are bigger than $0.040 \mathrm{~m}$, and Orientation 3 is better when the axial sizes are lower than $0.040 \mathrm{~m}$.
In Figure 31, when samples' axial sizes are lower than $0.040 \mathrm{~m}$, the CEC values of three orientations are close to each other; thus the PAE and COV values should be taken into consideration. Under this situation, if a higher PAE value is needed, samples should be placed in Orientation 1 and Orientation 2, and if a lower COV value is needed, samples should be placed in Orientation 3. When samples' axial sizes are bigger than $0.040 \mathrm{~m}$, Orientation 3 is not recommended due to the lower PAE and higher COV. If there is a specific 


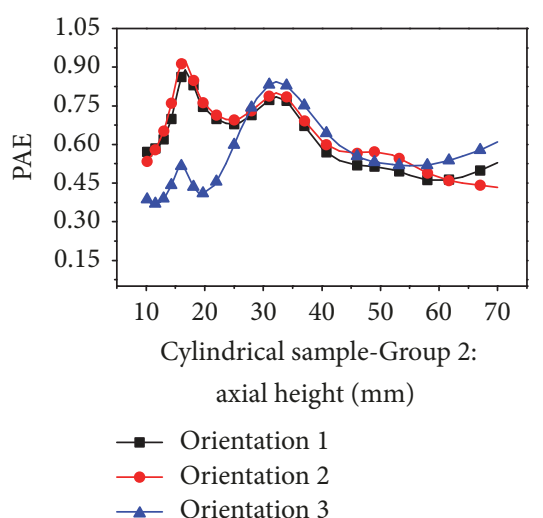

(a)

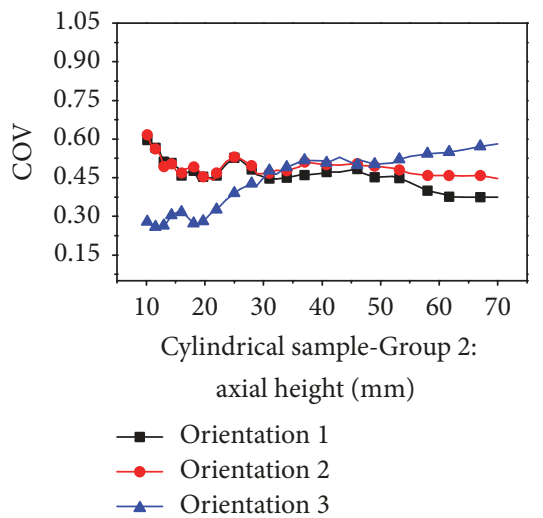

(d)

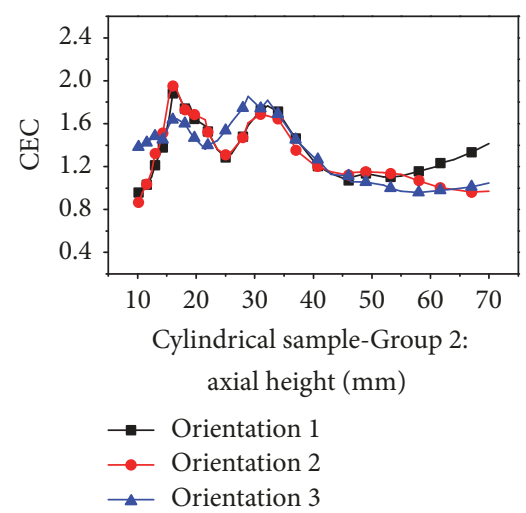

(g)

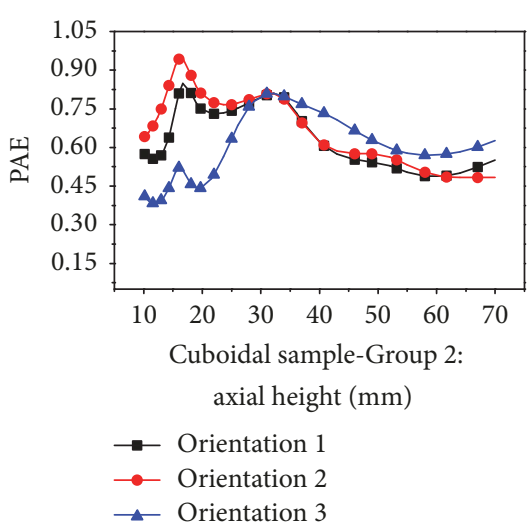

(b)

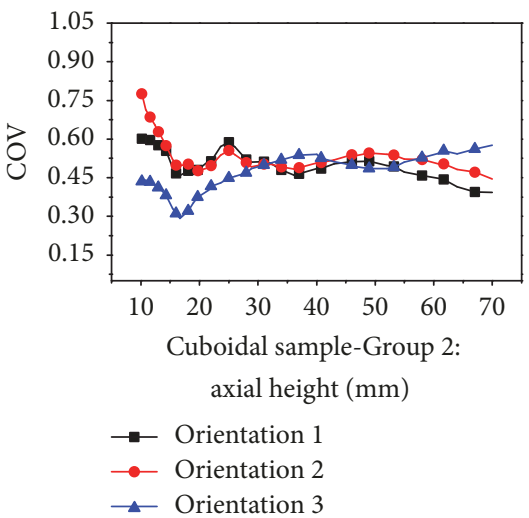

(e)

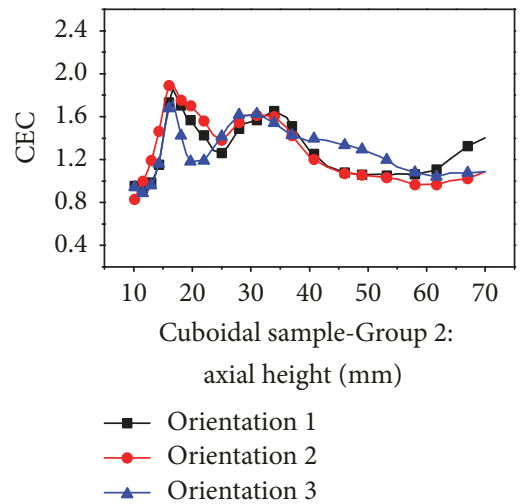

(h)

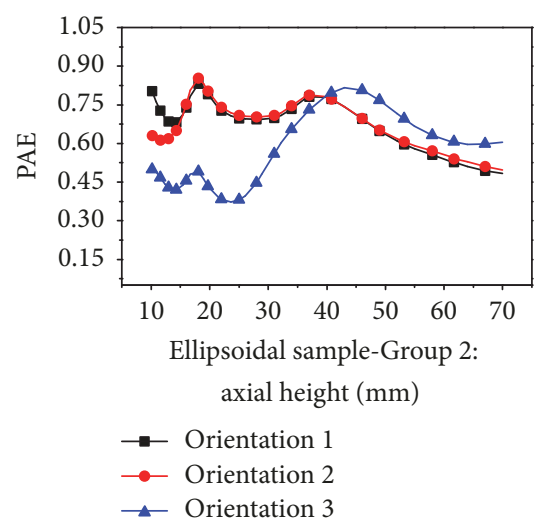

(c)

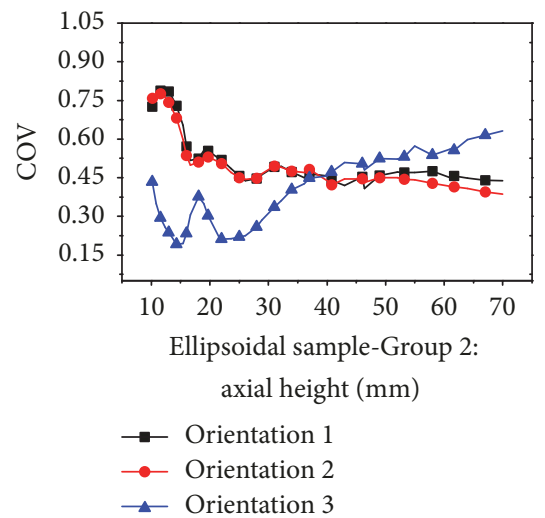

(f)

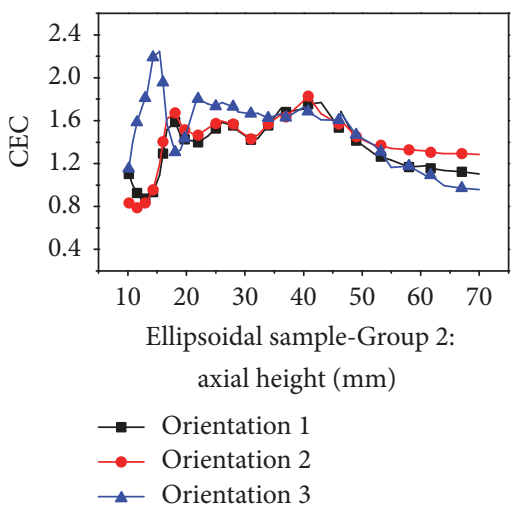

(i)

FIGURE 27: Same samples in different orientations, Group 2.

need for temperature uniformity, Orientation 2 is better; otherwise, Orientation 1 is better on this condition.

To sum up, in this section samples of the same shape are placed in different orientations, and the corresponding PAE, $\mathrm{COV}$, and CEC curves indicate that orientation influences the microwave process significantly. PAE, COV, and CEC values should be taken into consideration, when selecting orientations. From the study in this section, it can be concluded that when samples' axial sizes are bigger than $0.040 \mathrm{~m}$, Orientation 3 is the worst choice, Orientation 2 could be chosen only for the special needs of temperature uniformity, and Orientation 1 is the best choice due to the higher $\mathrm{PAE}$ and relatively lower temperature COV value. When samples' axial sizes are lower than $0.040 \mathrm{~m}$, Orientation 3 is recommended if temperature uniformity is important, and if microwave power absorption is more decisive, Orientation 1 and Orientation 2 are better.

\section{Conclusion}

Based on the above calculation results, it can be seen that, in the microwave heating cavity, samples with equal volume and 


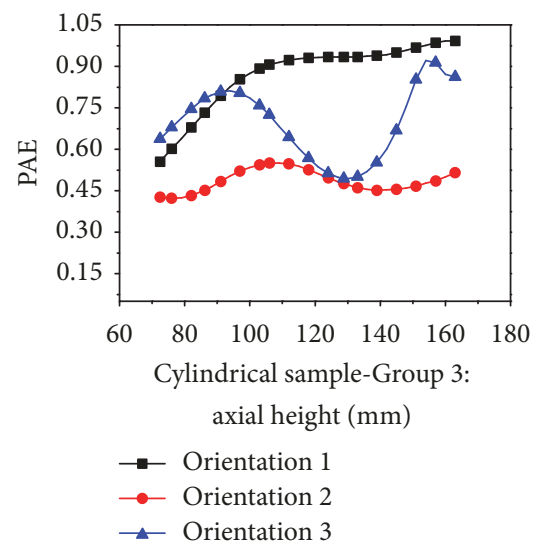

(a)

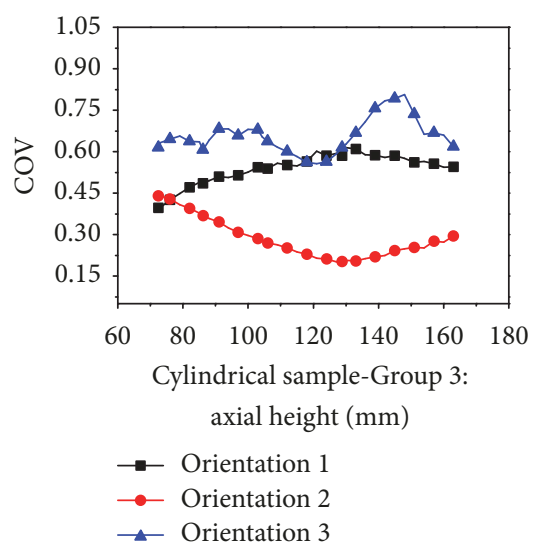

(d)

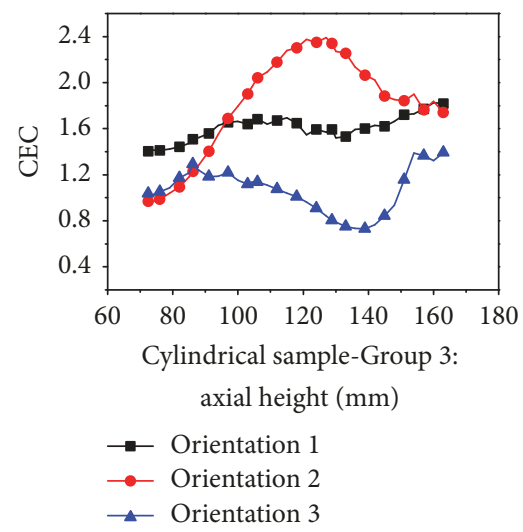

(g)

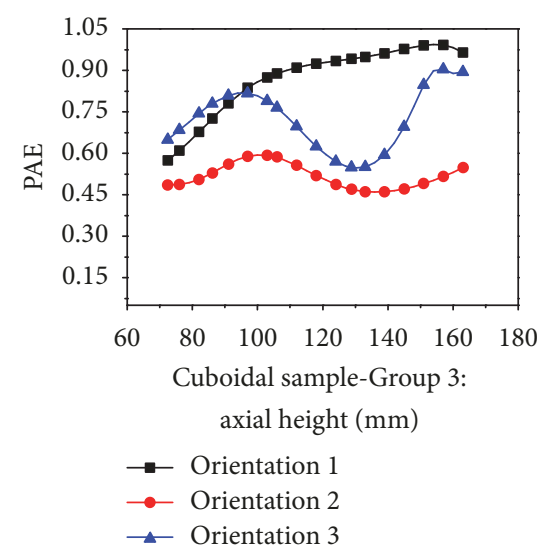

(b)

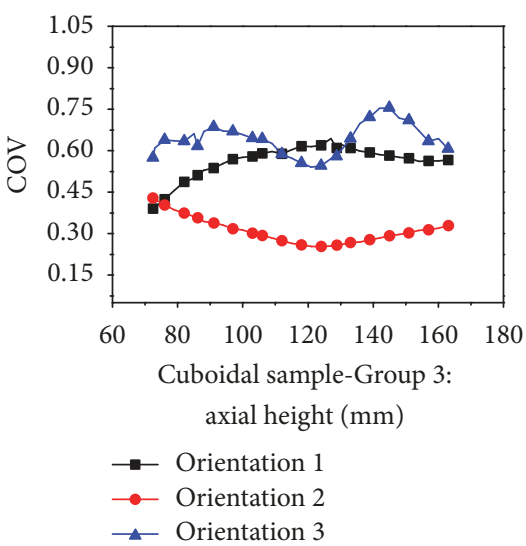

(e)

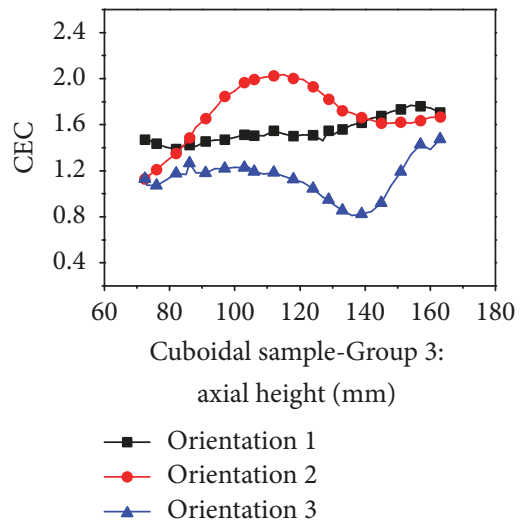

(h)

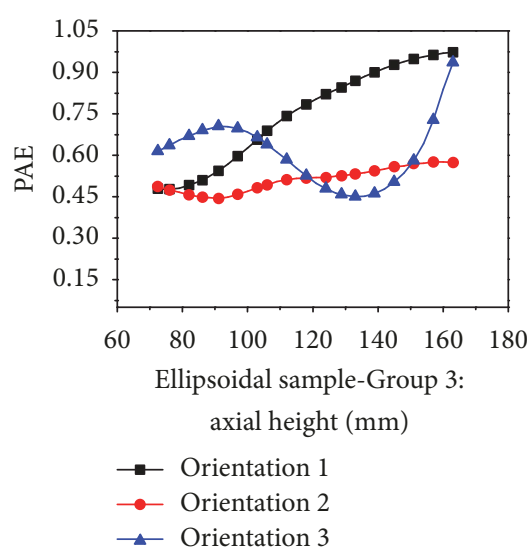

(c)

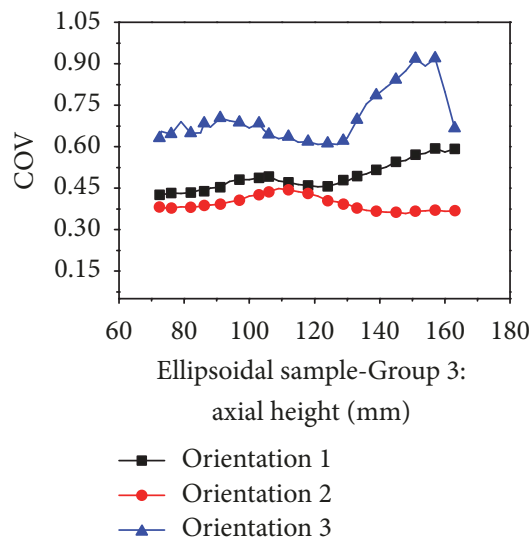

(f)

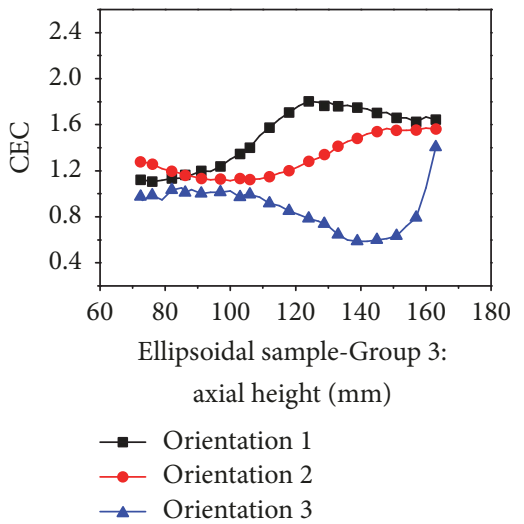

(i)

FIgURE 28: Same samples in different orientations, Group 3.

different shapes form hot spots at different positions in the center or near the surface of samples. Choosing proper input power can achieve the goal of high temperature distribution uniformity and low energy consumption. When the input power is larger than a certain value (which may change with sample's material), the final internal temperature uniformity tends to be constant.

The samples of spherical and cubic shapes can be classified into four groups according to the PAE and COV values as well as the locations of electromagnetic focusing and hot spots. For the small and big volume groups samples, spherical samples are better than the cubic ones. For the intermediate size samples, if temperature uniformity of samples is more important, spherical samples is the best choice, while if the decisive factor is the high microwave power absorption capability, cubic samples are recommended.

As for the isometric volume cylindrical, cuboidal, and ellipsoidal samples, they can be classified into three groups by the wave numbers and penetration numbers in the radial and axial directions. Intuitively, samples of Group 1 tend to be 


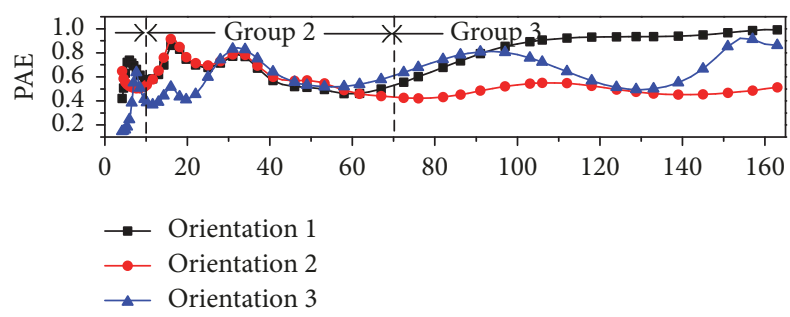

(a) Cylindrical sample: axial height $(\mathrm{mm})$

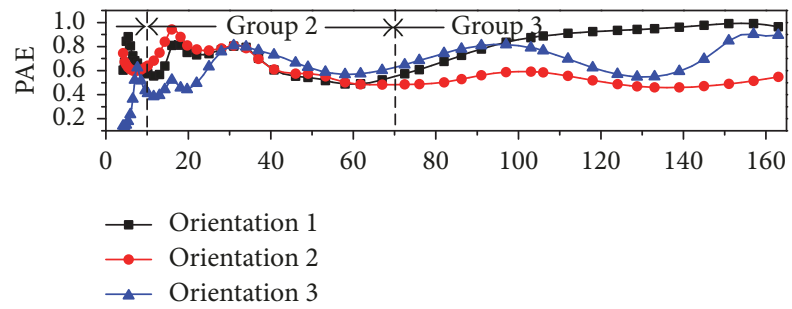

(b) Cuboidal sample: axial height $(\mathrm{mm})$

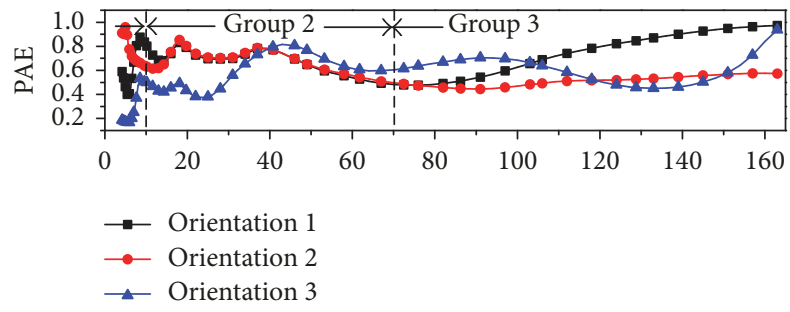

(c) Ellipsoidal sample: axial height $(\mathrm{mm})$

FIGURE 29: PAE of the same samples in different orientations.

more flat, while those belonging to Group 3 are more slender. Quantitatively, in Group 1, samples' radial and axial wave numbers and penetration numbers are $N_{p}^{r}>3, N_{w}^{a}>0.1$, and $N_{p}^{a}<3$. In Group 2, samples' radial and axial wave numbers and penetration numbers are $N_{w}>0.1$ and $N_{p}<3$. In Group 3 , samples' radial and axial wave numbers and penetration numbers are $N_{w}^{r}>0.1, N_{p}^{r}<3$, and $N_{p}^{a}>3$.

Microwave heating properties of samples of different shapes placed in the same orientation were elaborated by the means of intuitive figures and quantificational curves. PAE, COV, and CEC curves are used to comprehensively evaluate the usability of a sample. PAE, COV, and CEC curves of different shape samples in the same orientation have similar variation tendency. For samples belonging to Group 1, ellipsoidal shape sample is the optimal shape when placed in Orientation 1 and Orientation 2, and when the sample is placed in Orientation 3, cylinder is the best shape. For samples belonging to Group 2, when samples' axial sizes are lower than $0.040 \mathrm{~m}$, the effect of sample shape is small enough to be negligible; namely, every shape of samples could be chosen, and beyond this range ellipsoidal shape would be a better choice. For samples in Group 3, cylindrical samples are better when placed in Orientation 1 and Orientation 2, and when in Orientation 3, cuboid would be a better shape.

The PAE, COV, and CEC curves of samples of same shape placed in different orientations indicate that orientations affect the microwave process significantly. PAE, COV,

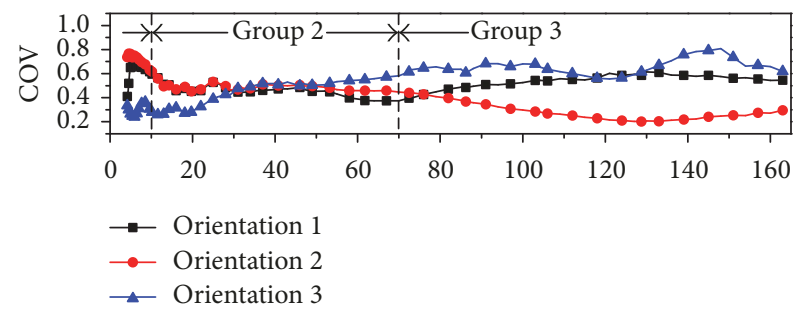

(a) Cylindrical sample: axial height ( $\mathrm{mm})$

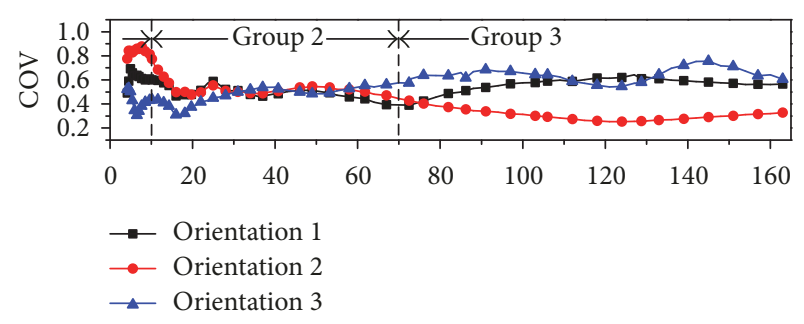

(b) Cuboidal sample: axial height $(\mathrm{mm})$

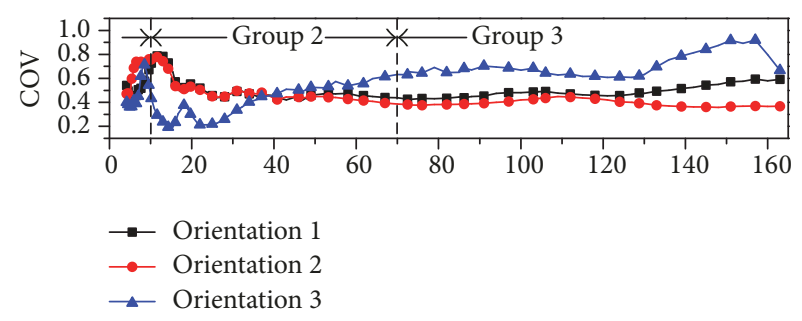

(c) Ellipsoidal sample: axial height $(\mathrm{mm})$

Figure 30: COV of the same samples in different orientations.

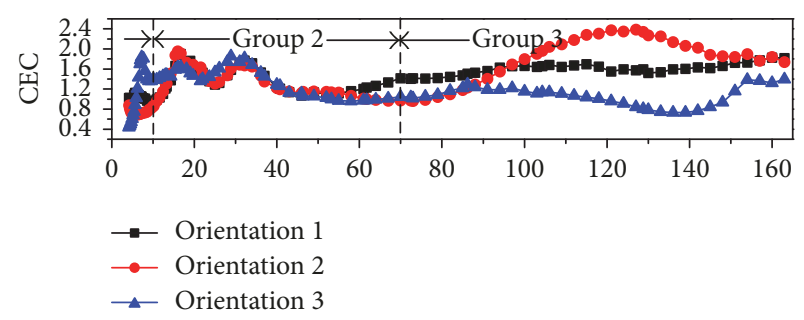

(a) Cylindrical sample: axial height $(\mathrm{mm})$

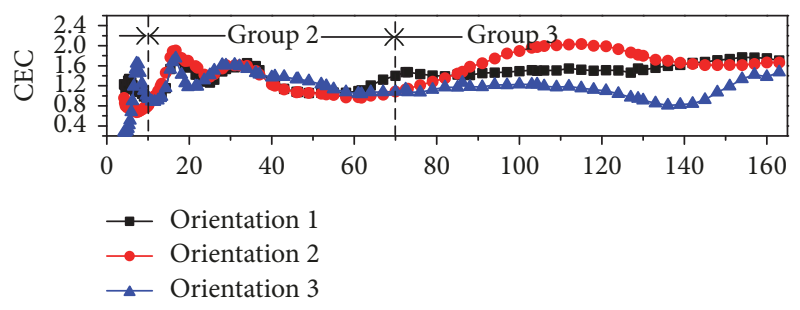

(b) Cuboidal sample: axial height $(\mathrm{mm})$

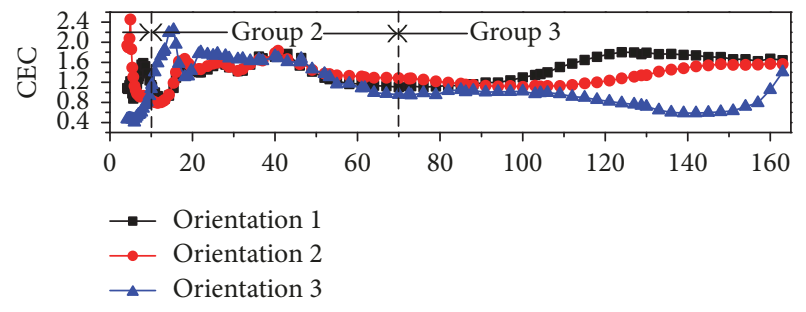

(c) Ellipsoidal sample: axial height (mm)

FIGURE 31: CEC of the same samples in different orientations. 
and CEC values should be taken into consideration when selecting orientations. When samples' axial sizes are bigger than $0.040 \mathrm{~m}$, Orientation 3 is the worst choice, Orientation 2 could be chosen only for the special needs of temperature uniformity, and Orientation 1 is the best choice due to the higher PAE and relatively lower temperature COV value. When samples' axial sizes are lower than $0.040 \mathrm{~m}$, Orientation 3 is recommended if temperature uniformity is important, and if microwave power absorption is more decisive, Orientation 1 and Orientation 2 are better.

\section{Conflicts of Interest}

The authors declare that there are no conflicts of interest regarding the publication of this paper.

\section{Acknowledgments}

This research was supported by the National Natural Science Foundation of China (Grant nos. 31371873, 31000665, 51176027, and 31300408).

\section{References}

[1] S. Begum and M. S. Brewer, "Physical, chemical and sensory quality of microwave-blanched snow peas," Journal of Food Quality, vol. 24, no. 6, pp. 479-493, 2001.

[2] W. H. Wu and D. A. Lillard, "Cholesterol and proximate composition of channel catfish (Ictalurus punctatus) fillets Changes following cooking by microwave heating, deep-fat frying, and oven baking," Journal of Food Quality, vol. 21, no. 1, pp. 41-51, 1998.

[3] S. B. M. S. Brewer and A. Bozeman, "Microwave and Conventional Blanching Effects on Chemical, Sensory, and Color Characteristics of Frozen Broccoli," Journal of Food Quality, vol. 18, no. 6, pp. 479-493, 1995.

[4] B. P. K. M. S. Brewer, K. Bharati, and A. K. Perry, "Microwave Blanching Effects on Chemical Sensory and Color Characteristics of Frozen Green Beans," Journal of Food Quality, vol. 17, pp. 245-259, 1994.

[5] T. P. W. H. S. Ramaswamy, "Temperature Distribution in Microwave Heated Food Models," Journal of Food Quality, vol. 15, pp. 435-448, 1992.

[6] J. F. Song, C. Q. Liu, X. Q. Jiang, and D. J. Li, “Quality Evaluation of Vacuum Microwave-Dried Immature Vegetable Soybean (Glycine Max [L.] Merr.)," Journal of Food Quality, vol. 38, no. 5, pp. 337-346, 2015.

[7] E. E. Abano, H. Ma, and W. Qu, "Influence of combined microwave-vacuum drying on drying kinetics and quality of dried tomato slices," Journal of Food Quality, vol. 35, no. 3, pp. 159-168, 2012.

[8] N. Therdthai, P. Ritthiruangdej, and W. Zhou, "Effect of Microwave Assisted Baking on Quality of Rice Flour Bread," Journal of Food Quality, vol. 39, no. 4, pp. 245-254, 2016.

[9] R. G. M. van der Sman and J. R. Bows, "Critical factors in microwave expansion of starchy snacks," Journal of Food Engineering, vol. 211, pp. 69-84, 2017.

[10] M. C. Law, E. L. Liew, S. L. Chang, Y. S. Chan, and C. P. Leo, "Modelling microwave heating of discrete samples of oil palm kernels," Applied Thermal Engineering, vol. 98, pp. 702-726, 2016.

[11] I. E. Marques, C. A. Bizzi, F. B. Lucion et al., "Are Infrared and Microwave Drying Suitable Alternatives for Moisture Determination of Meat Products?" Journal of Food Quality, vol. 39, no. 4, pp. 391-397, 2016.

[12] C. Conesa, L. Seguí, N. Laguarda-Miró, and P. Fito, "Microwaves as a pretreatment for enhancing enzymatic hydrolysis of pineapple industrial waste for bioethanol production," Food and Bioproducts Processing, vol. 100, pp. 203-213, 2016.

[13] S. Chandrasekaran, S. Ramanathan, and T. Basak, "Microwave food processing - a review," Food Research International, vol. 52, no. 1, pp. 243-261, 2013.

[14] R. R. Mishra and A. K. Sharma, "Microwave-material interaction phenomena: Heating mechanisms, challenges and opportunities in material processing," Composites Part A: Applied Science and Manufacturing, vol. 81, pp. 78-97, 2016.

[15] S. S. R. Geedipalli, V. Rakesh, and A. K. Datta, "Modeling the heating uniformity contributed by a rotating turntable in microwave ovens," Journal of Food Engineering, vol. 82, no. 3, pp. 359-368, 2007.

[16] M. S. Venkatesh and G. S. V. Raghavan, "An overview of microwave processing and dielectric properties of agri-food materials," Biosystems Engineering, vol. 88, no. 1, pp. 1-18, 2004.

[17] H. W. Yang and S. Gunasekaran, "Comparison of temperature distribution in model food cylinders based on Maxwell's equations and Lambert's law during pulsed microwave heating," Journal of Food Engineering, vol. 64, no. 4, pp. 445-453, 2004.

[18] K. G. Ayappa, H. T. Davis, E. A. Davis, and J. Gordon, "Analysis of microwave heating of materials with temperature-dependent properties," AIChE Journal, vol. 37, no. 3, pp. 313-322, 1991.

[19] C. A. Balanis, Advanced Engineering Electromagnetics, John Wiley \& Sons, Inc., New York, NY, USA, 1989.

[20] T. Basak, M. Bhattacharya, and S. Panda, "A generalized approach on microwave processing for the lateral and radial irradiations of various Groups of food materials," Innovative Food Science and Emerging Technologies, vol. 33, pp. 333-347, 2016.

[21] J. Chen, K. Pitchai, D. Jones, and J. Subbiah, "Effect of decoupling electromagnetics from heat transfer analysis on prediction accuracy and computation time in modeling microwave heating of frozen and fresh mashed potato," Journal of Food Engineering, vol. 144, pp. 45-57, 2014.

[22] T. Basak, "Role of various elliptical shapes for efficient microwave processing of materials," AIChE Journal, vol. 53, no. 6, pp. 1399-1412, 2007.

[23] H. Zhu, T. Gulati, A. K. Datta, and K. Huang, "Microwave drying of spheres: Coupled electromagnetics-multiphase transport modeling with experimentation. Part I: Model development and experimental methodology," Food and Bioproducts Processing, vol. 96, pp. 314-325, 2015.

[24] M. Bhattacharya, T. Basak, and S. Sriram, "Generalized characterization of microwave power absorption for processing of circular shaped materials," Chemical Engineering Science, vol. 118, pp. 257-279, 2014.

[25] M. U. H. Joardder, M. A. Karim, and C. Kumar, "Multiphase transfer model for intermittent microwave-convective drying of food: considering shrinkage and pore evolution," International Journal of Multiphase Flow, vol. 95, pp. 101-119, 2017. 
[26] J. Zhang, Y. Luo, C. Liao et al., "Theoretical investigation of temperature distribution uniformity in wood during microwave drying in three-port feeding circular resonant cavity," Drying Technology, vol. 35, no. 4, pp. 409-416, 2017.

[27] X. L. Hu, X. Q. Yang, and G. Z. Jia, "Effective optimization of temperature uniformity and power efficiency in two-ports microwave ovens," The Journal of Microwave Power and Electromagnetic Energy, vol. 48, no. 4, pp. 261-268, 2014.

[28] D. Luan, Y. Wang, J. Tang, and D. Jain, "Frequency Distribution in Domestic Microwave Ovens and Its Influence on Heating Pattern," Journal of Food Science, vol. 82, no. 2, pp. 429-436, 2017.

[29] A. K. D. V. Rakesh, M. H. G. Amin, and L. D. Hall, "Heating uniformity and rates in a domestic microwave combination oven," Journal of Food Process Engineering, vol. 32, pp. 398-424, 2007.

[30] Y. D. Hong, B. Q. Lin, H. Li, H. M. Dai, C. J. Zhu, and H. Yao, "Three-dimensional simulation of microwave heating coal sample with varying parameters," Applied Thermal Engineering, vol. 93, pp. 1145-1154, 2016.

[31] H. Chen, M. Zhang, Z. Fang, and Y. Wang, "Effects of Different Drying Methods on the Quality of Squid Cubes," Drying Technology, vol. 31, no. 16, pp. 1911-1918, 2013.

[32] M. Bhattacharya and T. Basak, "On the analysis of microwave power and heating characteristics for food processing: Asymptotes and resonances," Food Research International, vol. 39, no. 10, pp. 1046-1057, 2006.

[33] F. Chen, A. D. Warning, A. K. Datta, and X. Chen, "Susceptors in microwave cavity heating: Modeling and experimentation with a frozen pie," Journal of Food Engineering, vol. 195, pp. 191-205, 2017.

[34] C. Kumar, Modelling Intermittent Microwave Convective Drying (IMCD) of Food Materials [Doctor of Philosophy, Chemistry, Physics and Mechanical Engineering], Science and Engineering Faculty Queensland University of Technology, Australia, 2015.

[35] L. Zhou, V. M. Puri, R. C. Anantheswaran, and G. Yeh, "Finite element modeling of heat and mass transfer in food materials during microwave heating - Model development and validation," Journal of Food Engineering, vol. 25, no. 4, pp. 509529, 1995.

[36] The Microwave Processing of Foods, Woodhead Publishing Limited and CRC Press LLC, USA, 2005.

[37] M. Bhattacharya and T. Basak, "A comprehensive analysis on the effect of shape on the microwave heating dynamics of food materials," Innovative Food Science and Emerging Technologies, vol. 39, pp. 247-266, 2017.

[38] T. Gulati, H. Zhu, A. K. Datta, and K. Huang, "Microwave drying of spheres: Coupled electromagnetics-multiphase transport modeling with experimentation. Part II: Model validation and simulation results," Food and Bioproducts Processing, vol. 96, pp. 326-337, 2015.

[39] H. Zhang and A. K. Datta, "Heating concentrations of microwaves in spherical and cylindrical foods: Part Two: In a cavity," Food and Bioproducts Processing, vol. 83, no. 1 C, pp. 1424, 2005. 


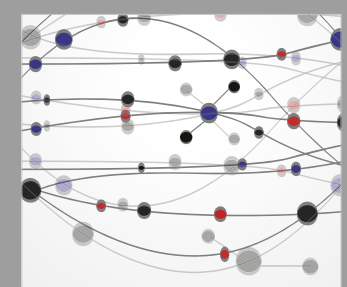

The Scientific World Journal
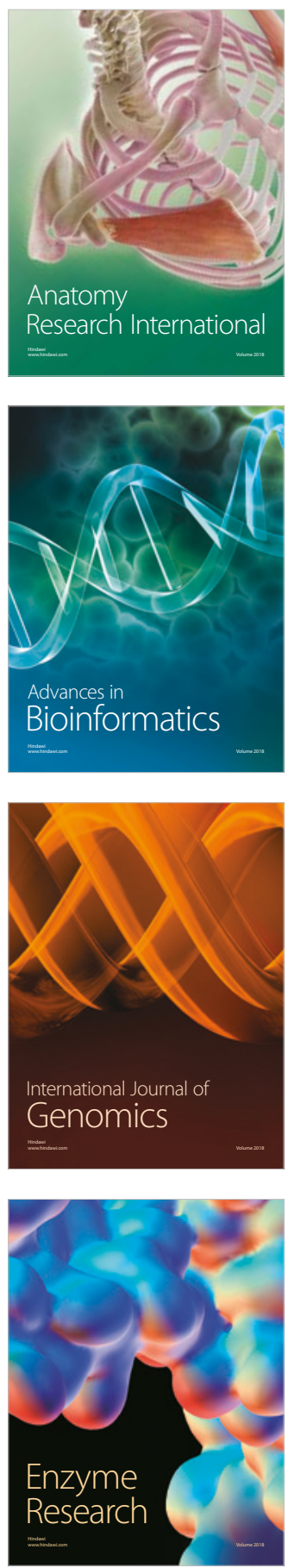
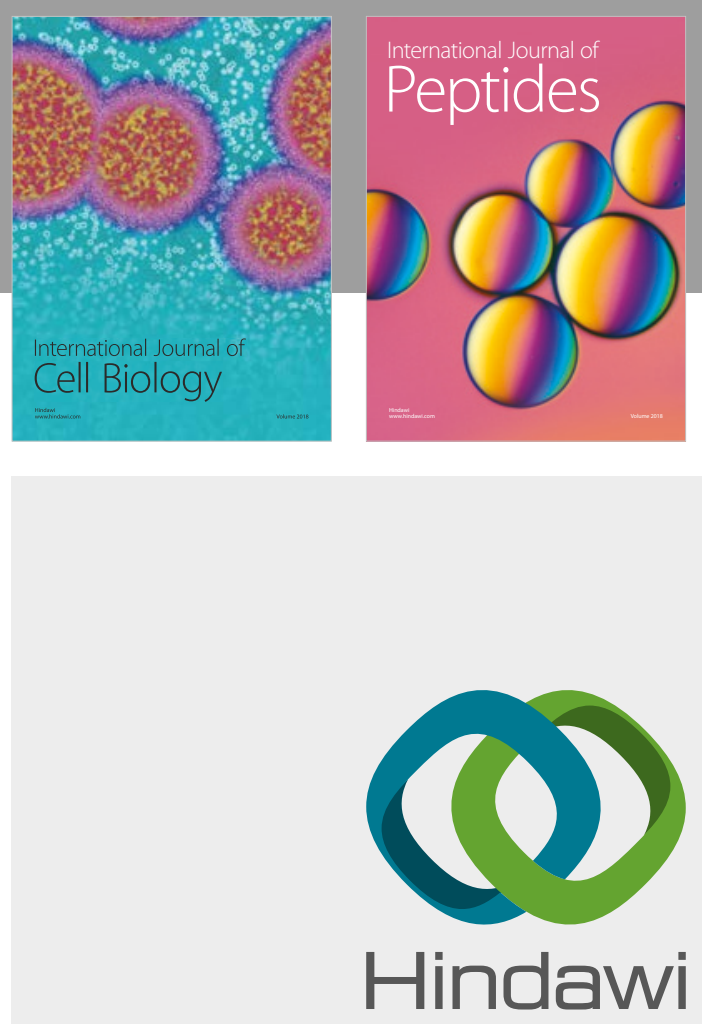

Submit your manuscripts at

www.hindawi.com
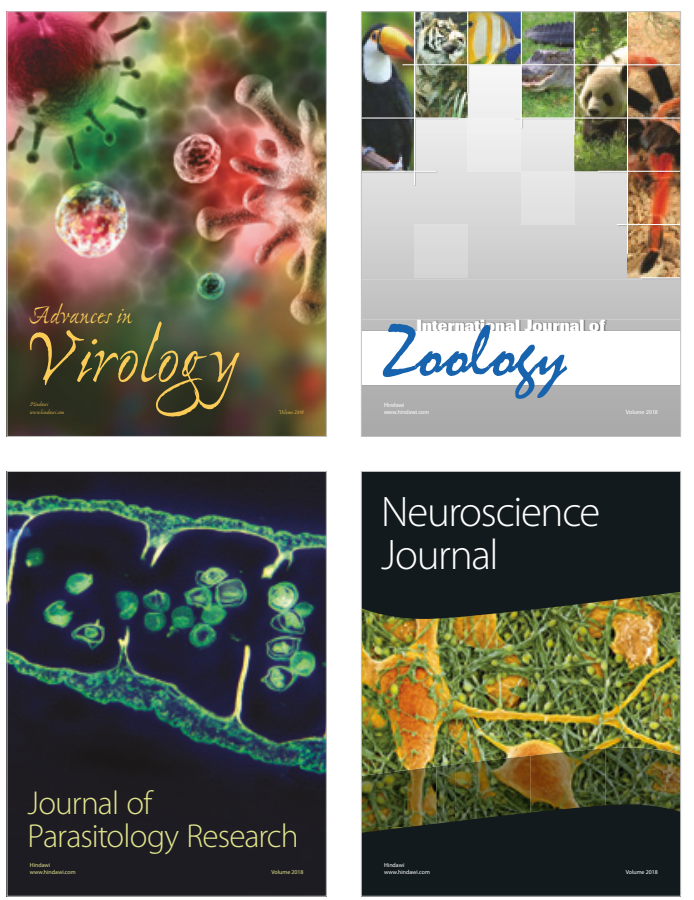
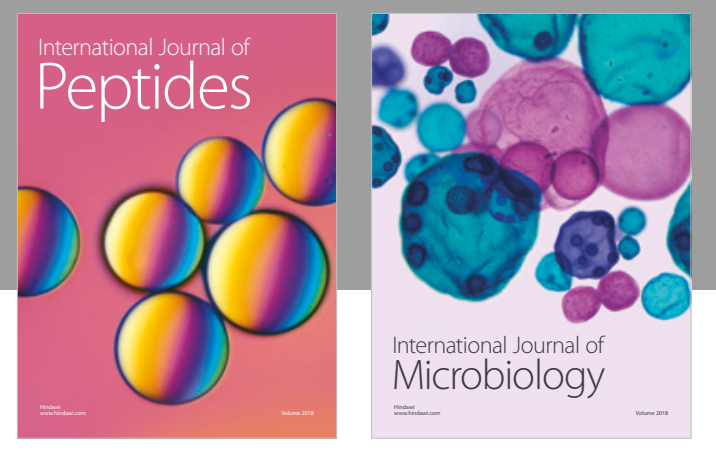

nternational Journal of Microbiology
Journal of
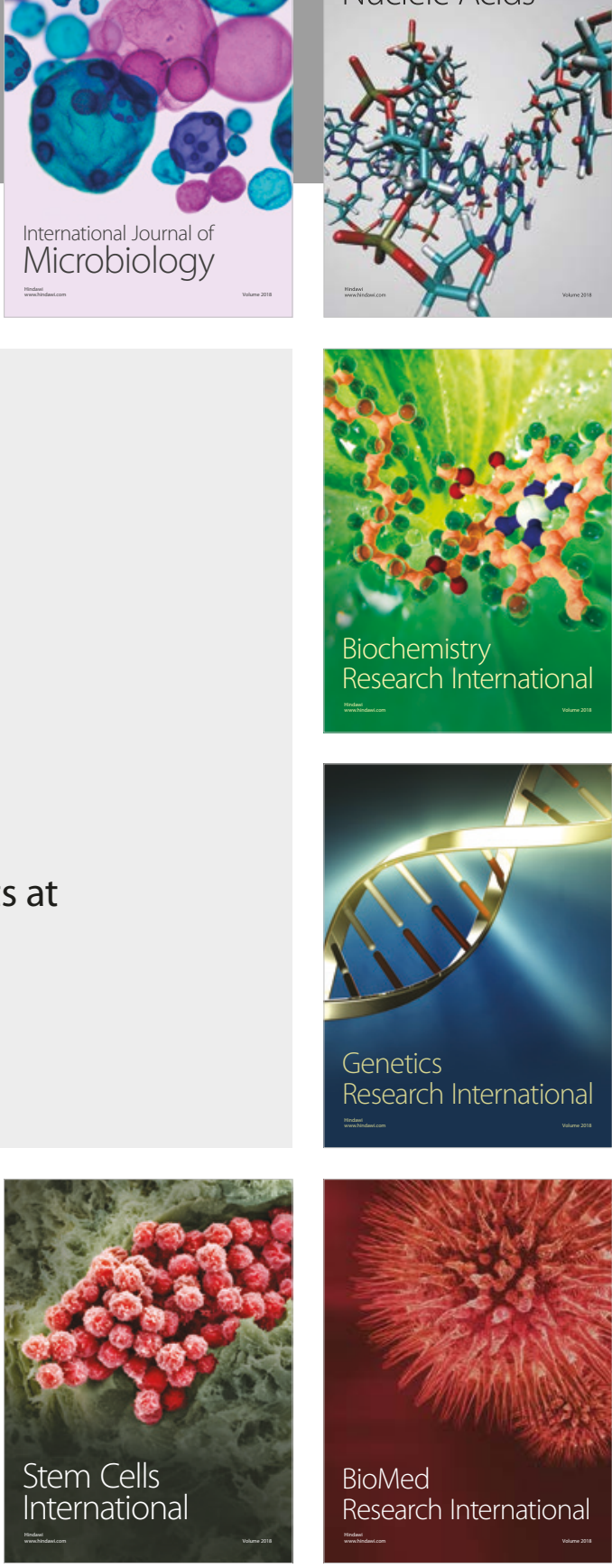
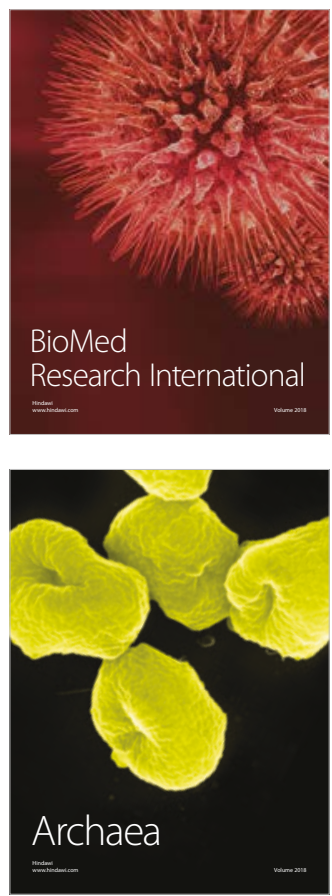